\title{
Contracting with Repeated Moral Hazard and Private Evaluations
}

\author{
William Fuchs*
}

September 6, 2005

\begin{abstract}
A repeated moral hazard setting in which the Principal privately observes the Agent's output is studied. It is shown that there is no loss from restricting the analysis to contracts in which the Agent is supposed to exert effort every period, receives a constant efficiency wage and no feedback until he is fired. The optimal contract for a finite horizon is characterized, and shown to require burning of resources. These are only burnt after the worst possible realization sequence and the amount is independent of both the length of the horizon and the discount factor $(\delta)$. For the infinite horizon case a family of fixed interval review contracts is characterized and shown to achieve first best as $\delta \rightarrow 1$. The optimal contract when $\delta<<1$ is partially characterized. Incentives are optimally provided with a combination of efficiency wages and the threat of termination, which will exhibit memory over the whole history of realizations. Finally, Tournaments are shown to provide an alternative solution to the problem.
\end{abstract}

\section{Introduction}

There are many environments in which the Principal might privately observe a signal of the Agent's output or performance. For example, if an analyst prepares a research report for his boss, both can see how many pages the report has, but how good or valuable does the boss find the report? This is his private information. The Principal might also privately receive reports from a client regarding the Agent's performance. In teamwork environments, the supervisor might elicit confidential reports from co-workers to better assess an employee's performance. Some procurement relationships also naturally fit this framework. For example, if a supplier ships goods across the Atlantic, the condition in which those goods are received may be privately observed by the buyer. Our goal in this paper

\footnotetext{
*I'm particularly indebted to Andy Skrzypacz and Tom Sargent for their valuable advice and suggestions. I thank Manuel Amador, Heski Bar-Isaac, Simon Board, Vinicius Carrasco, Ignacio Esponda, Narayana Kocherlakota, Ed Lazear, Jon Levin, Gustavo Manso, Paul Milgrom, Tomasz Sadzik, Yuliy Sannikov, Ilya Segal, Ennio Stacchetti and Pierre-Olivier Weill for their useful comments. Support from the B.F Haley and E.S Shaw Fund at Stanford University and the Mariscal the Ayacucho Fellowship is gratefully acknowledged.
} 
is to characterize optimal contractual arrangements in such environments with a moral hazard problem on the Agent's side and private information on the Principal's side.

We show in this paper how many features observed in labor markets - such as wage compression, involuntary unemployment, efficiency wages, review periods and tournaments- show up as alternatives to overcome the Agent's moral hazard when the Principal monitors the output privately.

In a related paper, MacLeod (2003) analyzed a one-shot model of a Principal who privately observes the performance of an Agent (who, in turn, first privately chooses his effort level). We extend MacLeod's analysis to games of arbitrary finite length and infinite horizons. For the finite horizon case, we reinforce MacLeod's results that incentives cannot be provided with a balancedbudget contract. Burning resources is necessary in order to simultaneously provide incentives for the Agent to exert effort and for the Principal to report output truthfully. The intuition for this result is that although we have to punish the Agent for bad outcome realizations, we cannot let the Principal benefit from it. Otherwise, he would want to lie and claim the outcome was low.

We characterize the optimal contract for a finite horizon and show that money burning will take place only at the end of the game and only if the Principal observes the worst output sequence. Note that this implies that it is optimal to pool all periods together before an evaluation. This is not due to the strength of the statistical test used to determine deviations. It derives instead from the fact that in the optimal contract, the only binding incentive constraint for the Agent is the one that guarantees he will exert effort in the first period. Therefore, once incentives are provided for the Agent no to shirk in the first period, we can get him to work the rest of the periods without incurring any additional informational costs. ${ }^{1}$

In the second part of the paper we consider the case when the horizon is infinite. One motivation to study this case is that it allows us to endogenize money burning through termination. Termination destroys the remaining surplus that could have been generated by the relationship. Explicitly studying how value is destroyed in the repeated game also raises several important issues that were not present in the finite horizon model. The fact that there is no 'last' period and that all the burning can no longer take place at the end of the game introduces new and important aspects to the problem. The question of the optimal timing for the release of information no longer has a straightforward solution. Even if the Principal sends no explicit messages to the Agent, the simple fact that he has not been fired provides the Agent with some information about the outcome realizations. This adds a series of new concerns when determining the optimal firing decisions.

From a thecnical perspective, this is an infinitely repeated game with private monitoring. Unfortunately, such games lack a tractable recursive structure. There is no equivalent to the tools

\footnotetext{
${ }^{1}$ This is closely related to the notion of "reusability of punishments" introduced in Abreu, Milgrom and Pearce (1991).
} 
developed by Abreu, Pearce and Stacchetti (1990) or Sannikov (2004) applicable to these types of games. Hence, characterizing the equilibrium value set (or even a given equilibrium) poses a great challenge. $^{2}$

In light of these difficulties, Levin (2003), who first analyzed the infinite horizon problem, restricted his attention to contracts with the "Full Review Property". This property restricts the analysis to equilibria in which the Principal truthfully reveals the outcome to the Agent every period. Hence, the Principal does not retain any private information from period to period. We show that these contracts are generally suboptimal, especially, when players are patient. We expand the allowable class of contracts by studying $T$-period review contracts: the Principal reviews the Agent's performance only at predetermined fixed intervals of length $T{ }^{3}$ The length of the review is in turn endogenously determined. We are able to establish that these contracts are asymptotically efficient: as the players become very patient, we can obtain per period payoffs arbitrarily close to the first best payoffs (the corresponding contracts have longer-and-longer review periods as $\delta \rightarrow 1$ ). This result cannot be achieved within the family of contracts with the "Full Review Property".

The next step is to analyze arbitrary contracts. First, we show that in order to characterize the equilibrium value set, we can restrict our attention to a specific class of contracts. This class entails the Agent exerting effort every period, receiving a fixed efficiency wage and no bonuses nor feedback on his past performance until he is fired.

These results resonate well with the evidence presented by Baker et al. (1994) that only $5 \%$ of workers claim to receive performance pay in the form of commissions or piece rate contracts and that only $25 \%$ claim to have bonuses based on subjective measures of performance. Also consistent with our results is Prendergast (1999) who documents the reluctance of managers to rate employees, especially when it impacts compensation.

Then we use a variation approach to provide further characterization of an optimal contract. This method consists in showing that if a given contract does not have certain properties, we can modify it slightly to obtain a weak improvement. This allows us to sidestep some of the difficulties associated with the absence of a tractable recursive representation; in particular, the fact that arbitrarily complex deviations by the Agent must be considered. ${ }^{4}$ This technique allows us to construct a partial ordering over histories and show that the termination decisions exhibit memory. Outcomes from past periods affect termination decisions for the whole future. Therefore, optimal contracts cannot be replicated by short-term contracts.

\footnotetext{
${ }^{2}$ See Kandori (2002) for a discussion of the difficulties associated with the analysis of private monitoring games.

${ }^{3}$ The case $T=1$ would correspond to contracts with the Full Review Property.

${ }^{4}$ The main technical difficulty is that in general it is not sufficient to check only one-step deviations by the agent.
} 
Finally, we show that organizational design features such as hierarchies or tournaments could provide an alternative solution to the problem. For example, if the Principal had another Agent, a tournament could be set up and used to provide incentives without the need to burn any resources.

The best way to relate this paper to the more general literature on moral hazard problems is to classify the models along the following two properties. Is the output verifiable by a third party? Is the output common knowledge between the Principal and the Agent? The first papers in contract theory such as Holmstrom (1979) and Shavell (1979) focused on the case in which the output was common knowledge and, more importantly, could be verified by a court of law. They studied static problems and their main concern was the risk sharing vs. incentives trade-off. In many circumstances output measures fail to be veriable by the courts. This problem was first analyzed Bull (1987) and MacLeod and Malcomson (1989). They showed that if the performance is common knowledge, continuation values in long term relationships could be used to provide incentives. Pearce and Stacchetti (1998) further showed how the verifiable and non-verifiable measures of performance could be combined in optimal contracts. The typical examples cited in the literature of situations in which output cannot be verified by the courts generaly involve complex output measures such as the quality of teaching or customer care. It is natural to think that the assumption of common knowledge over performance might also fail in these types of situations. Lifting this assumption leads to the work of MacLeod (2003), Levin (2003) and this paper.

This paper is also related to the literature on efficiency wages. The use of efficiency wages in macro models such as Shapiro and Stiglitz (1984) has been criticized for lacking proper microeconomic foundations. We provide such foundations and show that the contracts studied by Shapiro and Stiglitz (1984) are actually optimal in an environment where the Principal privately observes the Agent's output.

The rest of the paper is organized as follows: Section 2 presents the model. Section 3 shows that the analysis can be restricted to contracts in which the Principal pays a constant wage, the Agent exerts effort until he is fired and there is no communication. Section 4 studies the finite horizon problem. Section 5 extends the analysis to the infinite horizon. Possible organizational design solutions are studied in section 6. Section 7 explores extensions of the basic model to allow for random effort. Conclusions and suggestions for future research can be found in Section 8. Finally, ommited proofs can be found in Appendix B.

\section{The Model}

Two risk neutral players, an Agent and a Principal are matched at time zero and have the opportunity to trade at dates $t \in\{0,1, \ldots, T\}$. We will consider both the case where $T$ is finite and $T=\infty$. 
At $t=0$, the Principal and the Agent agree on a compensation schedule and a termination policy. ${ }^{5}$ Part of this agreement is determined by a long term contract $\omega$ signed at time zero. Its terms depend only on verifiable information that is enforceable by a third party. The rest of the agreement corresponds to a perfect Bayesian equilibrium (a self-enforcing contract) of the post-contractual repeated game $\Gamma_{\omega}$ we now describe.

The figure below depicts the order of play in each period of the game while the Agent is still employed. Informally, the timing is as follows: first, the Agent chooses whether to exert effort or not, conditional on his information. Next, the Principal privately observes the output realization $y_{t}$ and a random variable $\phi_{t}$. He then chooses a message $m_{t}$ to send to the Agent. This message is verifiable and implies a bonus $b_{t}$ he must pay to the Agent. The Principal also pays a base wage $w_{t}$ that is not contingent on output but can be contingent on a public randomization device $x_{t}$. Finally, simultaneous decisions are made in every period on whether to continue the relationship into the future or not. ${ }^{6}$

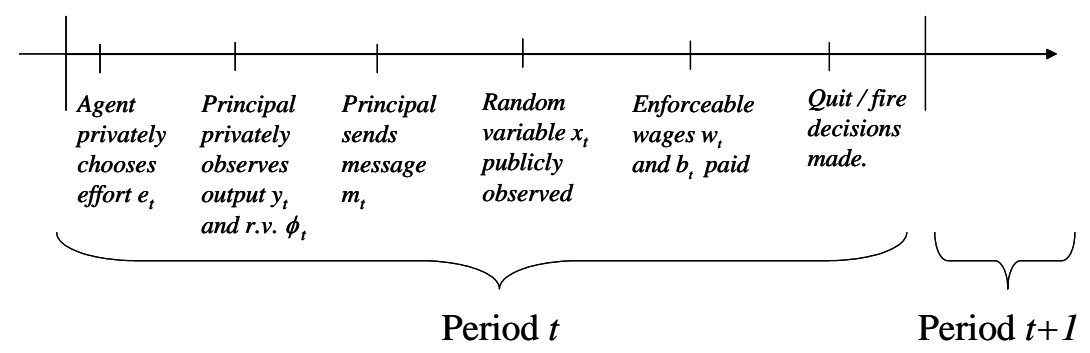

Given the particular information structure of the game we will define three different histories. First, we have the set of verifiable histories $H_{t}^{V}$ where $t$ denotes the length of the history. A typical element of this set is $h_{t}^{V}=\left\{m^{t}, k^{t}, \mathcal{A}^{t}, x^{t}\right\}$. Superscripts are used to denote sequences of realizations and subscripts are used to denote a particular realization. $k_{t}$ is an indicator function that equals one if the Agent decides to stay in the job at time $t$. Similarly, $\mathcal{A}_{t}$ is an indicator function that equals one if the Principal decides to continue employing the Agent at time $t$. The exogenous public randomization device $x_{t} \sim U[0,1]$ is independent of all other elements of the game and identically distributed over time. Next, we have the set of Agent's private histories $H_{t}^{A}$. A typical element in this set is $h_{t}^{A}=\left\{h_{t}^{V}, e^{t}\right\}$. Finally, we have the set of Principal's private histories $H_{t}^{P}$. A typical element in this set is $h_{t}^{P}=\left\{h_{t}^{V}, y^{t}, \phi^{t}\right\}$. Where $\phi_{t} \sim U[0,1]$ is independent of all other elements of the game and identically distributed over time. For all of the histories defined above we take the convention that $h_{-1}^{j}=\emptyset$.

\footnotetext{
${ }^{5}$ The way the surplus is divided is not important for the analysis so we won't model it explicitly. We think about it as the outcome of some efficient bargaining.

${ }^{6}$ The assumption that these decisions are simultaneous could be easily modified without affecting the results.
} 
We can now formally define an enforceable contract $\omega \in \Omega$ as two sequences of functions: base wages and bonuses. Base wages $w_{t}$ are random functions of all the verifiable history except the history of messages:

$$
w_{t}:\left(H_{t-1}^{V} / M^{t-1}\right) \times[0,1] \rightarrow \mathbb{R} \text { for } t=0, \ldots, T .^{7}
$$

Note that this formulation of the wages allows for severance pay. Bonuses are additionally functions of the messages sent by the Principal:

$$
b_{t}: H_{t-1}^{V} \times M_{t} \times[0,1] \rightarrow \mathbb{R}^{+} \text {for } t=0, \ldots, T .
$$

The assumption that $b \geq 0$ is without loss of generality since we could always rescale $\left\{w_{t}\right\}$; also, without loss of generality we assume that for all $t$ there is some $m_{t}$ such that $b\left(h_{t-1}^{V}, m_{t}, x_{t}\right)=0 .^{8}$ This is useful because we want to allow the Principal to send messages at no cost, for example if those messages contain no information.

Each period if still employed, the Agent has a choice of exerting effort or not, $e_{t}: H_{t-1}^{A} \rightarrow\{0,1\} .{ }^{9}$ We assume no effort is costless and exerting effort is costly. Formally, we denote the cost of effort by $c(e)$ where, $c(0)=0$ and $c(1)=c>0$.

Output is also binary, $y_{t} \in Y_{t}=\{L, H\}$ and is privately observed by the Principal. Its realizations are stochastic and depend on the level of effort in the following way:

\begin{tabular}{|l|l|c|}
\hline$P(y \mid e)$ & $y=L$ & $y=H$ \\
\hline$e=0$ & $1-q$ & $q$ \\
\hline$e=1$ & $1-p$ & $p$ \\
\hline
\end{tabular}

We assume that effort increases the likelihood of a high outcome:

$$
1>p>q>0 \text {. }
$$

We use the common support assumption since it facilitates some of the discussion, in particular when considering off equilibrium behaviour. The cases $p=1$ and $q=0$ are discussed later.

The expected output conditional on no effort is zero. ${ }^{10}$ Lastly, the per period expected surplus from exerting effort will be denoted by $s$ and assumed to be strictly positive:

$$
\mathbb{E}[y \mid e=1]-c=s>0 .
$$

\footnotetext{
${ }^{7}$ The $\times[0,1]$ stands for the public randomization device $x_{t}$.

${ }^{8}$ Let $\hat{b}$ be the minimun bonus for all possible messages at time $t$. Let $\tilde{w}_{t}=w_{t}+\hat{b} \forall w_{t}$ and $\tilde{b}\left(h_{t-1}^{V}, m_{t}, x_{t}\right)=$ $b\left(h_{t-1}^{V}, m_{t}, x_{t}\right)-\hat{b} \forall b_{t}$

${ }^{9}$ We consider mixed strategies for the Agent in Section 7.

${ }^{10}$ This is not purely a normalization since it implies that no effort no pay is identical to termination in terms of the surplus generated.
} 
Upon observing the realization of $y_{t}$ and $\phi_{t}$, the Principal sends a public and verifiable announcement $m_{t}$, where $m_{t}: H_{t-1}^{P} \times Y_{t} \times[0,1] \rightarrow M_{t}$. The set $M_{t}$ is the algebra generated by $Y^{t}{ }^{11}$ That is, the Principal can choose to reveal only partially or not reveal at all the history of outcomes to the Agent.

The last event in each period is the simultaneous decision whether to continue the relationship or terminate it. Termination occurs if at least one of the parties decides to terminate the relationship. The Agent's decision whether to stay or not is denoted $k_{t}$ where, $k_{t}: H_{t-1}^{A} \times e_{t} \times x_{t} \times M_{t} \rightarrow\{0,1\}$. The Principal offers the Agent to remain employed with probability $a_{t}$ where, $a_{t}: H_{t-1}^{P} \times Y_{t} \times$ $M_{t} \times[0,1] \rightarrow[0,1] .{ }^{12}$ If termination occurs both parties receive their outside options which are normalized to zero. ${ }^{13}$ If a relationship has been terminated, the parties never trade again (the Agent exerts no more effort for the Principal) only severance pay transfers may still take place.

Let $\sigma_{\omega}^{A}=\left[\left\{e_{t}\right\},\left\{k_{t}\right\}\right]$ denote the Agent's strategy conditional on having signed contract $\omega$. Similarly let $\sigma_{\omega}^{P}=\left[\left\{m_{t}\right\},\left\{a_{t}\right\}\right]$ denote the Principal's strategy conditional on having signed contract $\omega$. We will use $\sigma_{\omega}=\left(\sigma_{\omega}^{A}, \sigma_{\omega}^{P}\right)$ to denote a strategy pair. The full strategies for the players have an additional element $\chi^{i} i=A, P$ which denotes the choice of accepting a given contract $\omega$ with the self-enforcing agreement (to be defined in the next section) that $\sigma_{\omega} \in \Sigma_{\omega}^{A} \times \Sigma_{\omega}^{P}$ will be played in the continuation game. $\chi^{i}: \Omega \times \Sigma_{\omega}^{A} \times \Sigma_{\omega}^{P} \rightarrow\{0,1\}$. The complete strategy profile for each player is $\sigma^{i}=\left[\chi^{i}, \sigma_{\omega}^{i}\right]$. Denote by $\sigma$ a strategy profile pair $\left(\sigma^{A}, \sigma^{P}\right)$ of the whole game.

We use $\delta \in(0,1)$ to denote the discount factor which is assumed to be the same for both players. The Agent maximizes the expected discounted flow of wages minus the effort cost.

$$
V=\mathbb{E} \sum_{t=0}^{T} \delta^{t}\left(w_{t}+b_{t}-c\left(e_{t}\right)\right)
$$

The Principal maximizes the expected discounted value of output $y_{t}$ net of the wages. ${ }^{14}$

$$
F=\mathbb{E} \sum_{t=0}^{T} \delta^{t}\left(y_{t}-w_{t}-b_{t}\right)
$$

If the players follow a strategy profile $\sigma$ we can define the values for the players from starting the game with a history $h_{t-1}^{P}$ as follows:

\footnotetext{
${ }^{11}$ Alternatively: $m_{t}: H_{t-1}^{P} \times Y_{t} \times[0,1] \rightarrow \Delta M_{t}$. The set $M_{t}$ is the algebra generated by $Y^{t}$ and $\Delta M_{t}$ denotes the simplex over $M_{t}$.

${ }^{12}$ Note, that we allow for mixed termination strategies for the principal but not for the agent. This is shown in Section 3 not to be a restricitve assumption.

${ }^{13}$ We could embed this model into a matching model such as that in Shapiro and Stiglitz (1984). What we need is for there to be quasi rents perceived when matched.

${ }^{14}$ This formulation implicitly rules out the possibility that part of the wage paid could be destroyed before it is received by the Agent. We relax this assumption in Section 4.
} 
For the Agent:

$$
V_{t}\left(h_{t-1}^{P}, \sigma\right)=\mathbb{E}\left[\sum_{j=t}^{T} \delta^{(j-t)}\left(w_{j}+b_{j}-c\left(e_{j}\right)\right) \mid h_{t-1}^{P}, \sigma\right],
$$

and for the Principal:

$$
F_{t}\left(h_{t-1}^{P}, \sigma\right)=\mathbb{E}\left[\sum_{j=t}^{T} \delta^{(j-t)}\left(y_{j}-w_{j}-b_{j}\right) \mid h_{t-1}^{P}, \sigma\right] .
$$

If the Players are following $\sigma$ then the Principal knows both $F_{t}$ and $V_{t}$. Note that if the Agent deviates, the Principal will be mistaken on his evaluations of $F$ and $V{ }^{15}$ The Agent knows neither since they depend on the Principal's private information. He forms and expectation of $V_{t}$ based on his information and the strategies being played.

$$
v\left(h_{t-1}^{A}, \sigma\right)=\mathbb{E}\left[V_{t}\left(h_{t-1}^{P}, \sigma\right) \mid h_{t-1}^{A}, \sigma\right]
$$

\subsection{Equilibrium Definition}

An equilibrium of the post contractual game $\Gamma_{\omega}$ consists of:

A pair of strategies $\left[\sigma_{\omega}^{A}, \sigma_{\omega}^{P}\right] \in \Sigma_{\omega}^{A} \times \Sigma_{\omega}^{P}$ and a pair of posterior beliefs about the other player's type $\mu^{A}\left(h_{t}^{P} \mid e^{t}\right), \mu^{P}\left(h_{t}^{A} \mid y^{t}\right) \cdot{ }^{16}$

Definition 1 Best responses:

A strategy $\sigma_{\omega}^{A} \in \Sigma_{\omega}^{A}$ is a best response to $\sigma_{\omega}^{P}$ after history $h_{t-1}^{A}$ if:

$$
\sigma_{\omega}^{A} \in \arg \max _{\tilde{\sigma}_{\omega}^{A} \in \Sigma_{\omega}^{A}} v\left(h_{t-1}^{A}, \tilde{\sigma}_{\omega}^{A}, \sigma_{\omega}^{P}\right) .
$$

Similarly, a strategy $\sigma_{\omega}^{P} \in \Sigma_{\omega}^{P}$ is a best response to $\sigma_{\omega}^{A}$ after history $h_{t-1}^{P}$ if:

$$
\sigma_{\omega}^{P} \in \arg \max _{\tilde{\sigma}_{\omega}^{P} \in \Sigma_{\omega}^{P}} F\left(h_{t-1}^{P}, \sigma_{\omega}^{A}, \tilde{\sigma}_{\omega}^{P}\right) .
$$

Definition 2 A profile of strategies $\sigma_{\omega}^{*}=\left[\sigma_{\omega}^{* A}, \sigma_{\omega}^{* P}\right]$ and beliefs $\mu^{A}, \mu^{P}$ form a Perfect Bayesian Nash equilibrium of $\Gamma_{\omega}$ if and only if:

- $\sigma_{\omega}^{* A}$ is a best response to $\sigma_{\omega}^{* P}$ after every history $h_{t-1}^{A}$ and $\sigma_{\omega}^{* P}$ is a best response to $\sigma_{\omega}^{* A}$ after every history $h_{t-1}^{P}$ given the beliefs $\mu^{A}, \mu^{P}$.

- The beliefs $\mu^{A}, \mu^{P}$ are consistent with $\sigma_{\omega}^{*}$ and updated using Baye's rule, when possible.

Additionally, an equilibrium for the complete game requires that individual rationality constraints for signing the employment contract $\omega$ at time zero are satisfied.

\footnotetext{
${ }^{15}$ As we will explain later this is one of reasons why in general it won't be sufficient to only consider one step deviations for the Agent.

${ }^{16}$ The opponents type is defined by his private information.
} 


\section{Equilibrium Properties}

In this section we provide some general properties for any equilibrium $\left(\omega, \sigma_{\omega}^{*}\right)$. First, we show in Proposition 1 that all equilibria require surplus to be destroyed if in a given period the Agent was supposed to exert effort but a low outcome realization is observed. Next, we show that in order to analyze the possible equilibrium values we can concentrate in a sufficient class of contracts. In particular, for any equilibrium contract $\left(\omega, \sigma_{\omega}^{*}\right)$ there exists a payoff equivalent contract in which the Agent exerts effort every period and he is paid a constant wage (no bonuses) until he is fired. Furthermore, it is sufficient to focus on contracts in which the Principal does not send any informative messages to the Agent.

The following definitions facilitate our analysis.

Definition 3 (Continuation Values) $V_{t}^{+}\left(\left\{h_{t-1}^{P}, y_{t}\right\}, \sigma\right)$ is the promised continuation value to the Agent in period $t$ after $e_{t}$ has been exerted and the Principal has observed $y_{t}$. Similarly, $F_{t}^{+}\left(\left\{h_{t-1}^{P}, y_{t}\right\}, \sigma\right)$ is the principal's continuation value after observing and consuming $y_{t}$. Formally:

$$
\begin{gathered}
V_{t}^{+}\left(\left\{h_{t-1}^{P}, y_{t}\right\}, \sigma\right)=\mathbb{E}\left[w_{t}+b_{t}+\sum_{j=t+1}^{T} \delta^{(j-t)}\left(w_{j}+b_{j}-c\left(e_{j}\right)\right) \mid\left\{h_{t-1}^{P}, y_{t}\right\}, \sigma\right] \\
F_{t}^{+}\left(\left\{h_{t-1}^{P}, y_{t}\right\}, \sigma\right)=\mathbb{E}\left[-y_{t}+\sum_{j=t}^{T} \delta^{(j-t)}\left(y_{j}-w_{j}-b_{j}\right) \mid\left\{h_{t-1}^{P}, y_{t}\right\}, \sigma\right] .
\end{gathered}
$$

The Agent can only form an expectation of this value:

$$
v_{t}^{+}=\mathbb{E}\left[V_{t}^{+}\left(\left\{h_{t-1}^{P}, y_{t}\right\}, \sigma\right) \mid\left\{h_{t-1}^{A}, e_{t}\right\}, \sigma\right]
$$

Definition 4 Given $\sigma_{\omega}$ and a history $h_{t-1}^{V}$ such that $e\left(h_{t-1}^{V}, \sigma_{\omega}\right)=1$, we say that $\sigma_{\omega}^{A}$ is incentive compatible with respect to effort for the Agent (ICA) after history $h_{t-1}^{V}$ iff:

$$
\left(\begin{array}{l}
\mathbb{E}\left[V_{t}^{+}\left(\left\{h_{t-1}^{P}, y_{t}\right\}, \sigma_{\omega}\right) \mid\left\{h_{t-1}^{A}, e_{t}\right\}, \sigma_{\omega}^{A}, \sigma_{\omega}^{P}\right]- \\
\mathbb{E}\left[V_{t}^{+}\left(\left\{h_{t-1}^{P}, y_{t}\right\}, \sigma_{\omega}\right) \mid\left\{h_{t-1}^{A}, e_{t}\right\}, \tilde{\sigma}_{\omega}^{A}, \sigma_{\omega}^{P}\right]
\end{array}\right) \geq c,
$$

for all $\tilde{\sigma}_{\omega}^{A}$ such that $\tilde{\sigma}_{\omega}^{A} \equiv \sigma_{\omega}^{A}$ for all $\tau<t$ and $\tilde{e}\left(h_{t-1}^{V}\right)=0 .{ }^{17}$

\footnotetext{
${ }^{17}$ Note that $\tilde{\sigma}_{\omega}^{A}$ can include arbitrary future deviations both in the effort and in the termination, but agrees with equilibrium until $t-1$.
} 
Definition 5 Given $\sigma^{A}$ and $\omega$ we say $\sigma_{\omega}^{P}$ is incentive compatible for the Principal (ICP) after history $\left\{h_{t-1}^{P}, y_{t}\right\}$ iff:

$$
F_{t}^{+}\left(\left\{h_{t-1}^{P}, y_{t}\right\}, \sigma\right) \geq F_{t}^{+}\left(\left\{h_{t-1}^{P}, y_{t}\right\}, \sigma^{A}, \tilde{\sigma}^{P}\right)
$$

for all $\tilde{\sigma}_{\omega}^{P}$ such that $\tilde{\sigma}_{\omega}^{P} \equiv \sigma_{\omega}^{P}$ for all $\tau<t .^{18}$

\section{Surplus Destruction on Equilibrium Path}

The destruction of value after a low outcome is necessary to have contracts in which the Agent's continuation value is contingent on the realization of outcome while leaving the Principal indifferent. This is stablishe in the following result which extends Proposition 2 in MacLeod (2003) to games of arbitrary horizon.

Proposition 1 Providing incentives for high effort requires that in expectation some surplus be destroyed if $e_{t}=1$ and $y_{t}=L$ i.e :

$$
\begin{aligned}
& \mathbb{E}\left[V_{t}^{+}\left(\left\{h_{t-1}^{P}, y_{t}=H\right\}, \sigma\right) \mid\left\{h_{t-1}^{A}, e_{t}=1\right\}, \sigma\right]+F_{t}^{+}\left(\left\{h_{t-1}^{P}, y_{t}=H\right\}, \sigma\right) \\
> & \mathbb{E}\left[V_{t}^{+}\left(\left\{h_{t-1}^{P}, y_{t}=L\right\}, \sigma\right) \mid\left\{h_{t-1}^{A}, e_{t}=1\right\}, \sigma\right]+F_{t}^{+}\left(\left\{h_{t-1}^{P}, y_{t}=L\right\}, \sigma\right) .
\end{aligned}
$$

When dealing with a risk-neutral agent in an environment in which output is verifiable a contract making the Agent the residual claimant could be written and it would deliver first best. Here those types of contracts cannot work because the Principal would always claim output was low. Furthermore, there is no contract that can deliver first best.

Corollary 1 The first best is not achievable for $\delta<1$.

Proof. First note that the unconstrained optimum has the Agent exerting effort every period. By Proposition 1 if the Agent is to exert effort, there must exist some value burning which is inefficient.

\section{Sufficient Class of Contracts}

Next we present one of our main results, Theorem 1, which

\footnotetext{
${ }^{18}$ In words, $\tilde{\sigma}_{\omega}^{P}$ is consistent with $\sigma_{\omega}^{P}$ up to $t-1$ but can include arbitrary future deviations both in the messages and in the termination.
} 
Theorem 1 Given any contract $\left(\omega, \sigma_{\omega}\right)$ that generates values $V$ and $F$, we can construct a payoff equivalent contract $\left(\hat{\omega}, \hat{\sigma}_{\omega}\right)$ with the following properties:

(i) The Agent receives a constant wage until he is fired and no bonuses.

(ii) The Agent exerts effort every period until he is fired.

(iii) The Principal gives no feedback (sends no messages) to the Agent.

This strong result provides a possible explanation for the high degree of wage compression observed. It shows that for any contract there is a payoff equivalent contract that eliminates the need for bonuses. Incentives for the agents to exert effort is instead provided via efficiency wages and the threat of termination. Also worth remarking is the fact that Principal need not give any informative feedback to the Agent about his performance.

The fact that efficiency wages and not bonuses are used to provide incentives when explicitly analyzing the infinite horizon model suggests that we should reconsider the results of MacLeod (2003) regarding the use of bonuses vs. efficiency wages as incentive devices. We will do this in the next section.

\section{The Finite Horizon Case}

In this section we first show that if we requiere budget balance there is no enforceable contract that can provide incentives for the Agent to exert effort. Having established this result we then characterize the optimal contract when money burning is allowed.

Proposition 2 If $T=1, \sigma_{\omega}^{*}$ is essentially unique and it has $e(\omega)=0$ and $m(y)$ such that $b(m)=0 .{ }^{19}$ Furthermore, for any finite $T$ the game has as a unique equilibrium the repetion of the equilibrium for when $T=1$ as a result of unravelling.

If there is no remaining surplus in the relationship, incentives for effort cannot be provided because there is no way to burn resources in case of failure. Hence, we cannot provide contingent pay for the Agent leaving the Principal indifferent as required by Proposition 1.

To get around these negative results, MacLeod (2003) considered the possibility of having the wage being paid by the Principal differ from that recieved by the Agent, the difference between the two being burnt. He motivates his use of money burning as a shortcut to account for conflict in organizations (inefficient play in the continuation game if the game were to be repeated) ${ }^{20}$ Actual money burning is also sometimes used. For example, some baseball teams have the ability to fine

\footnotetext{
19 "Essentially" since there could be more than one $m$ such that $b(m)=0$.

${ }^{20}$ MacLeod (2003 pp217): "These costs are generated when individuals either leave the relationship or carry out inefficient actions for several periods..."
} 
their players but the money collected from these fines is not pocketed by the club but rather given away to charity. ${ }^{21}$ For the remainder of the section we define $w^{P}$ and $w^{A}$ as, respectively, the wage paid by the Principal and the amount the Agent receives.

In order to characterize the optimal contract with money burning we first show that without loss of generality we can focus on contracts in which wages are only paid in the last period and the Agent is to exert effort every period.

Lemma 1 All payments and money burning can be done in the last period.

Lemma 2 For every contract with $V+F>0$ there is a payoff equivalent contract in which the Agent exerts effort in every period.

These results are important for two reasons. First, when no bounds are placed on the amount of money burning that is allowed, it is sufficient to look at contracts with no termination. Destruction of value, if necessary, can be done via money burning. Second, since wage payments and money burning can be left for the last period, the Agent can be kept completely uninformed of the interim realizations of output. In this case, the Agent's dynamic problem of deciding in which periods to exert effort is identical to a static multitasking problem.

We make use of the static multitasking representation to characterize a contract that minimizes the amount of resources burnt (that maximizes surplus). The amount of resources burnt after a outcome sequence $y^{T}$ will be denoted by $Z\left(y^{T}\right)$. We will use $P\left(y^{T} \mid \mathbf{e}\right)$ to denote the probability that outcome sequence $y^{T}$ is realized conditional on the column vector of effort choices e. ${ }^{22}$ We use $\mathbf{L}$ to denote a vector of low outcome realizations. Finally we define $\mathbf{c}$ as the row vector of effort costs where the $i^{\text {th }}$ element is given by $\delta^{i-1} c$.

Proposition 3 An optimal contract that provides incentives for the Agent to exert effort for $T+1$ periods satisfies:

$$
\begin{array}{ll}
w_{T}^{A}=w_{T}^{P}-Z & \text { if } y_{t}=L \forall t \\
w_{T}^{A}=w_{T}^{P} & \text { otherwise. }
\end{array}
$$

Where:

$$
Z=\frac{c}{\delta^{T}(p-q)(1-p)^{T}}
$$

\footnotetext{
${ }^{21}$ We thank Jon Levin for providing this example.

${ }^{22}$ We denote vectors in bold.
} 
and:

$$
\frac{\sum_{t=0}^{T} \delta^{t} s_{t}}{\delta^{T}} \geq w_{T}^{P} \geq \frac{\sum_{t=0}^{T} \delta^{t} c}{\delta^{T}}+\frac{(1-p) c}{(p-q)} .
$$

Furthermore, when $\delta=1$, this is the only optimal contract.

Although the form of optimal contract, characterized with a two-step function, appears to relate closely to that described in Proposition 6 of MacLeod (2003) it is worth pointing out that it provides a very different insight for pay compression than that observed in a one-shot game. The fact that money burning only occurs after the worst possible history is the combined result of two factors. First, note that burning money after a history that has $y_{t}=H$ for some $t$ is providing incentives for the Agent to shirk in period $t$. The only history that does not provide these perverse incentives is $y^{T}=\mathbf{L}$. Second, although burning period by period as failures occur would also be an alternative that does not provide incentives for shirking, it is not optimal. We might be tempted to think that determining burning on the joint outcome of two periods is benefitial since it reduces the likelihood of burning. Although the likelihood of punishment occuring effectively decreases, the amount to be burnt when it occurs is simultaneously and proportionally increasing, eroding away all the gains from the lower frequency of occurrence.

The actual reason for pooling periods together, which follows from Corollary 2 below, is that the expected cost to motivate effort is independent of the horizon. Therefore, it is optimal to pool all the periods together in order to pay this cost only once. This is closely related to the results by Abreu, Milgrom and Pearce (1991) on the reusability of punishments, in the sense that one threat of punishment is used to simultaneously deter the temptation to deviate in any period.

Corollary 2 The expected present value cost to motivate effort is given by $c \frac{(1-p)}{(p-q)}$. It is independent of both $T$ and $\delta$.

The intuition behind this Corollary is as follows. In the proof of the Propositon we have shown that the only binding constraint is the one for effort in the first period. Since, the cost of the first period's effort, is not discounted by $\delta$, varying $\delta$ has no effect. The invariance with respect to $T$ also follows from there only being one constraint binding in the optimal contract. Since using $Z^{*}$ once we provide incentives for the Agent not to shirk in the first period, the constraints of the remaining periods are automatically satisfied. As mentioned before, the likelihood that burning takes place decreases in $T$ but exactly offsetting this effect is the fact that, when required, there will be more burning with larger $T$. The intuition for this is that the less the Agent believes that the first period's outcome is pivotal in triggering the punishment, the harsher the punishment must be to prevent him from shirking.

Comparative static results on the expected present-value cost to motivate effort are straightforward and intuitive. First, the lower the cost of effort the lower the inefficiency. If it is not too 
costly for the Agent to exert effort, then it is easier to provide incentives. Next, the smaller the difference in the probabilities of success conditional on exerting or not effort $(p-q)$, the harder it is to provide incentives for the Agent. As $p-q$ gets smaller, the outcomes observed if he shirks are closer to the ones if he doesn't, hence there is little reward for his effort and therefore it is hard to provide incentives. On the other hand as $p$ increases the probability that the Agent fails in the first period if he exerts effort decreases and hence there will be less burning in expectation. When $p=1$ there is no inefficiency incurred since on the equilibrium path $y^{T}=\mathbf{L}$ is never observed. Comparing the inefficiency of the optimal contract relative to the first best we can see that the only other case in which this contract approximates first best is if both $\delta \rightarrow 1$ and $T \rightarrow \infty$. For all other cases, efficiency is bounded away from the first best. Intuitively this follows because the cost of inducing effort is independent of both $T$ and $\delta$ and as we take the value of the relationship to $\infty$ this cost becomes insignificant in relative terms.

Corollary 2 also implies that for there to be trade between the parties we need:

$$
\sum_{t=0}^{T} \delta^{t} s \geq c \frac{(1-p)}{(p-q)} .
$$

If the condition above is not met providing incentives for the Agent to exert effort would deliver a negative surplus. A potential resolution to this problem using tournaments is discussed in Section 6.

We have shown that for the construction of the optimal contract, communication was not necessary. The following Proposition strengthens this result by showing that any feedback reduces the achievable efficiency.

Proposition 4 (No Feedback) When $\delta=1$, the optimal contract is not implementable if the Principal sends informative messages to the Agent.

The intuition for the result is clear, revealing information to the Agent has no advantage and it increases the amount of incentive constraints the Principal needs to worry about. When $\delta<1$ since all but the first constraint are slack, the Principal could send some very uninformative messages to the Agent and still be able to implement the optimal contract. When $\delta=1$ on the other hand, there are no slack constraints and any information would reduce efficiency because the optimal contract would cease to be incentive compatible.

When we analyze the limit of the finite horizon economies as $T \rightarrow \infty$ the problem has no solution if we allow for arbitrary amounts of money burning. As $T \rightarrow \infty$, the optimal contract calls for burning infinite amounts infinitely far in the future. Therefore, we need to establish some bound on the amount of money that can be burnt. The most natural way we see to do this is to determine it 
endogenously. We achieve this objective by limiting the amount of value that can be burnt to the potential surplus in the relationship. Furthermore, as mentioned earlier, a motivation for the use of money burning has been the cost of conflicts between Agents and Principals. Leaving potential sabotages aside, the termination of a relationship seems to be a natural way in which to account for these costs. In the next section we show though that using money burning as a shortcut to represent the destruction of value in an infinitely repeated game is only appropriate when we focus on a class of contracts called T-Period Review contracts. When looking at the optimal equilibria of the infinitely repeated game several new issues arise that are not present in the finite horizon model. In particular, since now the Agent has to receive some information in finite time, we must take into account that the timing of punishments also induces the Agent to learn and potentially makes providing incentives harder in the future. Also, the Agent might have incentives to deviate in order to learn about the Principal's private information.

\section{The Infinite Horizon Case}

Analyzing the infinite horizon version of the game presents a great challenge. The reason is that this is a game with private monitoring and, as pointed out by Kandori and Matsushima (1998) and further discussed in Kandori (2002), private monitoring games lack a tractable recursive structure. ${ }^{23}$ Hence, characterizing the equilibrium value set of infinitely repeated Private Monitoring Games is an objective that has not yet been achieved. A further complication related to the lack of a recursive structure is that when analyzing a candidate equilibrium it is not in general sufficient to consider only one-step deviations. This hinders any attempt at solving the problem in a direct way using Lagrangean methods. For these reasons, most of the papers in the area have focused on proving Folk Theorems and there has been little or no progress in characterizing optimal equilibria for $\delta<<1$.

To avoid these difficulties, Levin (2003) focuses his analysis on equilibria with the 'Full Review Property'. This condition requieres that the Principal reveal to the Agent what he observed after each period. We relax this condition in Section 5.1 and consider a class of equilibria in which the Principal only reveals his private information at fixed intervals of length $T .{ }^{24}$ Furthermore, $T$ is endogenously determined. We label the contracts with this property $T$-period review contracts. We show that using these contracts we are able to truncate the private histories and give the problem a simple recursive representation. An important property of these contracts is that the attainable per-period average payoffs approximate first best as $\delta \rightarrow 1$.

\footnotetext{
${ }^{23}$ Kandori (2002) is the introductory article to a special edition of The Journal of Economic Theory on Private Monitoring Games.

${ }^{24}$ The case $T=1$ corresponds to the contracts with the Full Review Property studied by Levin (2003).
} 
Although these contracts are asymptotically optimal as $\delta \rightarrow 1$, they are generally not optimal for $\delta<<1$. The Principal could provide incentives at a lower cost if instead of forgetting the past history after the Agent moves into a new review period he kept an account of it. Once we allow for this the problem becomes much more complex. We lose the recursive representation and we can no longer rule out multiple deviations by the Agent. In Section 5.2 we build on our results from Section 3 characterizing further the optimal contract. We return in this section to the original model in which we do not allow for exogenous money burning.

\subsection{Review Contracts}

A T-period review contract can be described as follows: after the predetermined review length $T$, the Principal evaluates the Agent's performance. If the performance is favorable, the Agent is offered employment with a clear record at $T+1$. If performance is not satisfactory, then the Agent is fired with probability $\beta$. If he is not fired, he is also offered employment with a clear record at $T+1$. Thus the game is partioned into independent review periods.

Within the literature on private monitoring games, similar type of equilibria appear in Compte (1998) and Kandori and Matsushima (1998). These, as our own, are based on the original work by Abreu, Milgrom and Pearce (1991), who study the timing of information revelation in an imperfect public monitoring environment. The models differ since in Abreu, Milgrom and Pearce (1991) the information is released by a third party and hence, there are no incentive compatibility considerations regarding the veracity of the reports in their analysis. Nonetheless, we observe that the potential for efficiency gains from the 'reusability of punishments' (attained by extending the length of the review) is still present. We exploit this and show that within this family of contracts we can obtain arbitrarily efficient contracts as $\delta \rightarrow 1$.

The $T$-period review contracts can also be thought of as a repetition of a $T$ period game with money burning, where there is a limited amount of money that can be burnt. Another simplification that the $T$-period structure gives us is that there are predetermined dates for the release of information. Recall that in the finite-horizon game there was no release of information untill the end, this of course cannot be true in an infinite horizon game. The question therefore arises of when is the best time to release information to the Agent. The longer the horizon the greater the efficiency, but the more that needs to be burnt if required. Since this amount is endogenously bounded, it in turn sets an endogenous bound on the review length.

We focus on a particular type of contract that pays a fixed wage every period $w=p H+(1-p) L$ while the Agent is employed. Every $T$ periods the Principal evaluates the Agent. If the output in all the $T$ periods under review has been low, he terminates the relationship with probability $\beta_{T}$. 
This type of contract is very convenient for tractability reasons, since, as we show in the following Lemma, we only need to check one incentive compatibility constraint for the Agent.

Lemma 3 With a T-period review contract, if it is not profitable for the Agent to deviate by exerting low effort in the first period, then there are no profitable deviations for the Agent. ${ }^{25}$

The IC constraint for the Agent in the $T$-period review contract boils down to:

$$
\delta^{T-1}(1-p)^{T-1}(p-q)(\delta \beta V) \geq c .
$$

Where the Agent's value is:

$$
V=\frac{S_{T}}{1-\delta^{T}\left(1-\beta(1-p)^{T}\right)}
$$

and $S_{T}$ denotes the present value of the surplus in $T$ periods:

$$
S_{T}=\sum_{t=0}^{T-1} \delta^{t} s .
$$

Note that the constraint ( $I C A T$ ) looks identical to the one we had for the one-period review case except for the first two terms in the left hand side. The first term simply reflects the discounting and the second is the probability that the Agent's decision to put effort in the first period is actually relevant. Furthermore, these terms are decreasing in $T$, tightening the constraint. Offsetting this effect is the fact that $V$ is increasing in $T$.

The constraint $(I C A T)$ can alternatively be expressed as follows:

$$
c+(1-p)^{T} \frac{(q-p)}{(1-p)} \delta^{T} \beta V \leq 0 .
$$

Expressed this way it closely resembles what we saw in Section 4. Except that now, the term $(\beta V)$ plays the role of $Z$, the amount of money burning.

Using the fact that $(I C A T)$ binds in the optimal contract, we can re-write $V$ as:

$$
V=\frac{S_{T}-\frac{c(1-p)}{(p-q)}}{1-\delta^{T}} .
$$

Defining the value in this way highlights the similarity to a repeated $T$-period money burning problem. It also shows why implementing the longest possible review length is optimal. ${ }^{26}$ The review length will be bounded by the need to have $\beta \leq 1$. We can solve for the optimal $\beta$ given $T$ :

\footnotetext{
${ }^{25}$ The proof, which is ommited, follows the same arguments as Part (iv) of Proposition 3.

${ }^{26}$ The $(I C A T)$ constraint allows us to conjecture that if we allowed for limited amounts money burning, both instruments would play the same role in the provision of incentives. Furthermore, that they would be used together to extend the review length as much as possible.
} 


$$
\beta^{*}=\frac{\left(1-\delta^{T}\right)}{\delta^{T}} \frac{c}{(p-q)(1-p)^{T-1}\left(S_{T}-\frac{c(1-p)}{(p-q)}\right)}
$$

A necessary condition for the implementability of a contract of length $T$ is that:

$$
S_{T}>\frac{c(1-p)}{(p-q)}
$$

This simply establishes that in period $T$ there is something positive to be burnt. Of course this alone is not sufficient since the exact amount to be burnt depends as well on all the other parameters. In particular note that as players are more patient longer and longer review lengths can be implemented. Furthermore, as long as the per period surplus is positive $(s>0)$ if players are very patient, then an inplementable contract always exists as shown in the following Lemma.

Lemma 4 In any relationship with positive surplus $s>0$ as we let $\delta \rightarrow 1$ there is some review length $T$ large enough such that high effort is implementable.

Definition 6 The inefficiency $\lambda_{T} \in[0,1]$ of an implementable contract, is defined by the value $V$ it achieves, relative to the value of First Best:

$$
\lambda_{T}=1-\frac{V}{V^{F B}}
$$

Conditional on the contract being implementable, the inefficiency $\lambda_{T}$ is given by:

$$
\lambda_{T}=\frac{c(1-p)}{(p-q)} \frac{1}{S_{T}}
$$

We can see from (3) that the same forces that were present in determining the inefficiency in the finite horizon play a role here. Additionally, the fact that we limit the amount of resources that can be burnt to the surplus in the relationship makes the inefficiency a function of the surplus that can be generated during the length of the review. As the per period surplus increases or as we are able to increase the review length, we are able to reduce the inefficiency. In particular, as $\delta \rightarrow 1$ we can make $\lambda_{T} \rightarrow 0$ since as $\delta \rightarrow 1$ we can take $T \rightarrow \infty$ and therefore $S_{T} \rightarrow \infty$. The Theorem below formalizes this result. 
Theorem 2 (Folk Theorem) For any $\varepsilon>0$ and $s>0$, there exists a $\delta^{*}<1$ such that for all $\delta>\delta^{*}$ we can construct a $T(\varepsilon, s)$ period review contract which is implementable and has less than $\varepsilon$ inefficiency.

We can relate this result back to the characterization of the optimal contract with money burning. As $\delta \rightarrow 1$ the future value of the relationship grows and hence, the constraint that having the amount of burning endogenously determined by the future surplus of the relationship becomes weaker and weaker. Hence, as it was the case for the finite horizon with $T \rightarrow \infty$, we want to drive the review time as far out in the future as possible and only terminate after the worst history.

Although the $T$-period review contracts are very convenient to analyze and asymptotically efficient, for $\delta<1$ they are generally not optimal. One exception is the special case $p=1$, in this case even the one period review contract achieves first best. This result can be related to Shapiro and Stiglitz (1984). They use one-period review contracts in which the Agent gets all the surplus to derive a theory for efficiency wages and involuntary unemployment. They don't prove that this type of contract is optimal. Instead, they claim that other types of contracts, such as having bond posting by the Agent, could be hard to implement because "the firm could have an incentive to claim the worker shirked to claim the bond". In this paper we rigorously show that the one-period review contract is actually optimal over all contracts for the special case they analyze.In the following Section we proceed to further characterize the unrestricted optimal contract for the case $\delta<1$.

\subsection{Partial Characterization of the Optimal Contract}

From Theorem 1 in Section 3 we know that the highest achivable surplus, can be obtained with a contract in which:

- The Agent exerts effort until he is fired.

- The Principal pays the Agent a constant wage equal to the expected output.

- No informative messages are sent to the Agent about his past performance.

As previously shown, this type of contract takes care of the Principal's incentive constraints. Incentives for the Agent are provided through the Principal's threat to terminate the relationship if the output sequence is indicative of cheating by the Agent. Optimality requires the minimum possible amount of termination that will provide incentives for the Agent to exert effort.

The Principal chooses a sequence of history dependent functions $a\left(h^{t}\right)$ that determine the probability with which the relationship is continued after history $h^{t}=\left\{y^{t}\right\}$. The Agent on the other hand 
chooses a sequence of effort choices $\left\{e_{t}\right\}_{t=0}^{\infty}$ that maximize his expected value. We use $h^{\tau} \subseteq h^{t}$ to denote initial subhistories of length $\tau$ of a longer history $h^{t}$. We use $p\left(h^{t}\right)$ to denote the probability that a given history is realized.

The objective is to maximize expected surplus conditional on the Agent exerting effort every period. Formally, the problem can be stated as follows:

$$
\begin{gathered}
V=\max _{\left\{a\left(h^{t}\right)\right\}} \sum_{t=0}^{\infty} s\left(\delta^{t}\left(\sum_{h^{t}} p\left(h^{t}\right) \prod_{h^{\tau} \subseteq h^{t}} a\left(h^{\tau}\right)\right)\right) \\
\text { s.t. }\left\{e_{t}=1\right\}_{t=0}^{\infty} \in \arg \max \sum_{t=0}^{\infty}\left(y^{e}-c\left(e_{t}\right)\right)\left(\delta^{t}\left(\sum_{h^{t}} p\left(h^{t} / e^{t-1}\right) \prod_{h^{\tau} \subseteq h^{t}} a\left(h^{\tau}\right)\right)\right)
\end{gathered}
$$

The following Proposition provides a partial characterization of the optimal contract using variational arguments. ${ }^{27}$ We use the notation $\left(h^{t}, h^{j}\right)$ to represent the history $h^{t+j}$ where first $h^{t}$ was observed and then $h^{j}$ was observed. When $h^{j}$ contains just one observation we will use $\left(h^{t}, H\right)$ or $\left(h^{t}, L\right)$.

Proposition 5 A sequence of continuation rules $\left\{a\left(h^{t}\right)\right\}_{t=0}^{\infty}$ that does not have the following properties, can be weakly improved upon.

(i) For all $h^{t}$, if $a\left(h^{t}, H\right)<1$ then $a\left(h^{t}, L\right)=0$.

(ii) For all $h^{t}, a\left(h^{t}, L\right) \leq a\left(h^{t}, H\right)$.

(iii) For all $h^{t} \times h^{j}$, if a $\left(h^{t}, H, h^{j}\right)<1$ then $a\left(h^{t}, L, h^{j}\right)=0$.

(iv) For all $h^{t} \times h^{j}, a\left(h^{t}, H, h^{j}\right) \geq a\left(h^{t}, L, h^{j}\right)$.

(v) For all $t$, if $a\left(L^{t}\right)>0$ then $a\left(h^{t}\right)=1$ for all $h^{t}$.

(vi) For all $h^{t}, V\left(h^{t}, H\right) \geq V\left(h^{t}, L\right)$.

(vii) For all $h^{t}$, if $a\left(h^{t}, L\right)=a\left(h^{t}, H\right)=0$ then $a\left(h^{t}\right)=0$.

\footnotetext{
${ }^{27}$ As mentioned before, the problem above is too complex to use Lagrangean methods.
} 
Altering any element of $\left\{a\left(h^{t}\right)\right\}_{t=0}^{\infty}$ impacts incentives in two ways. First, it affects the incentives of all previous periods through the effect the change has on the continuation values. Second, it impacts all future periods incentive constraints because the change affects the beliefs the Agent has about his past performance. Hence, the pertubations me make of the original contract are constructed in such a way that both future and past periods incentives are kept constant but incentives for the period under examination are relaxed.

Parts (i) \& (iv) of the the Proposition establish a partial ordering of the outcome histories. Essentially, for two histories $h^{t}$ and $\tilde{h}^{t}$, if $h^{t}$ element wise dominates $\tilde{h}^{t}$ (i.e. if for all $\tau \leq t$

$\left.y_{\tau} \geq \tilde{y}_{\tau}\right)$, then $a\left(h^{t}\right) \geq a\left(\tilde{h}^{t}\right)$. This result is further strengthened by parts (i) \& (iii) that show that if $a\left(h^{t}\right)<1$ then $a\left(\tilde{h}^{t}\right)=0$. Part (vii) states that an Agent who has no future would rather be fired immediately than kept for an additional period. This result would be natural if the Agent knew he had no future. On the other hand, when he doesn't know his standing, we could have thought that keeping him one more period could be useful because he generates one more period worth of surplus. This is shown not to be the case.

\section{Organizational Design: Optimal Contracts with a Third Party}

So far we have only considered the contractual possibilities between a single Agent and a single Principal. In this section we show that if there were other parties available, there could be more efficient ways in which to organize production. There are two organizational design solutions we would like to highlight. The first is the use of tournaments. The second is the role of hierarchies within an organization. Our goal in this section is not to provide a rigorous and exhaustive analysis of the topics discussed, but rather to highlight that our model can also help explain certain common features in organizations.

\subsection{Tournaments}

The role of tournaments as an incentive device for the agents has been largely studied in the literature starting with the seminal paper of Lazear and Rosen (1981). The literature on tournaments has generally assumed that output is contractable. By changing that assumption, this paper highlights a virtue of tournaments that, with the exception of Malcomson (1984), has not received much attention in the past. The tournament structure allows for contracts that provide a constant continuation value for the Principal for all output realizations and upward-sloping schedules for the Agents without burning any surplus.

Suppose the Principal can commit to give a prize $b$ to the best performing Agent every period (or randomize in case of a draw). This way the Principal has no more constraints since he has to 
give a prize to some player but is really indifferent about which Agent he gives it to. The Agents have incentives exert high effort in order to win the prize.

For example with two Agents, to provide incentives for them to exert high effort we need:

$$
\left(\frac{p-q}{2}\right) b-c \geq 0
$$

The term multiplying the bonus is by assumption positive hence we can always find a bonus big enough to provide incentives for the Agents to exert high effort. Note that this might require the base wage to be negative depending on how much of the surplus is captured by the Principal and how much by the Agents.

Tournaments themselves are in turn susceptible to other problems. Examples of these shortcomings include: the Agents colluding against the Principal or the Principal colluding with one of the Agents against the other. The Agents might also engage in unproductive activities to try to win over the Principal's favor or directly to try to undermine the other Agent's work. Therefore, we would expect to observe tournaments in environments in which the information problem is of more importance than these other concerns.

\subsection{Hierarchies:}

There are many potential explanations for the existence of hierarchies in organizations. In this section we point out that an additional advantage of such structures is that they serve to mitigate the incentives problems when the Principal observes output privately.

Suppose the Principal could hire an additional employee. This employee, the Supervisor, evaluates the Agent's performance and determines his wage. She, in turn, earns a fixed wage. Given that her wage is independent of the performance rating she assigns, the Agent believes she will report honestly and there will be no more need to destroy value. Of course, issues such as the Agent or the Principal trying to bribe the Supervisor could arise in this environment and would need to be prevented with appropriate devices. Also, a wage needs to be paid to the Supervisor reducing the surplus left for the Agent and Principal to share. The point, nonetheless, is that the use of hierarchical systems with those above determining the bonuses of those immediately below could be a way to address the combined incentives problems present when the Agent's output is privately observed by its Supervisor.

\section{Extensions: Stochastic Effort and Communication}

In the contracts we have analyzed so far, the Principal has been doing all the monitoring. The continuation values are conditional only on his observations. We might think there is potential to 
grant some monitoring power to the Agent. If the Agent follows a mixed strategy for effort, he would have private information on what the outcome distribution should look like. This information can then be compared to the Principal's output reports. We could conjecture that this might lead to an improvement. Although a complete analysis of these issues is left for future work we want to provide two results of interest illustrating whether this is or not the case.

We first prove a negative result showing that within the framework of our model and focusing on one-period review contracts it is optimal to have the Agent exert high effort deterministically.

Proposition 6 Allowing for contracts in which the Agent randomizes and the continuation values are determined as a function of the simultaneous announcements by the Agent and the Principal does not provide any improvement over the one-period review contract in which the Agent exerts high effort deterministically.

Proof. See Appendix A.

The intuition behind this negative result is that when the Agent mixes, the Principal actually has more incentives to claim $L$ instead of $H$. This follows from the fact that the Principal cannot be punished for claiming low output if effort was low. Therefore, when the Principal observes $H$ he knows there is some probability that the Agent had actually done low effort and hence that his lie will go unpunished. This intuition would seem to generalize when considering arbitrary contracts.

If instead the model allowed for an inefficiently high effort level, then things could work differently. Consider for simplicity the case in which if the Agent exerts a very high effort, then output is high for sure. Now, let the Agent mix between the efficient effort level and this higher effort level. The Principal is now more scared to declare $L$ when $H$ was realized. He knows that there is a chance that the Agent exerted the very high effort level and, that if this is the case, he would be caught lying if he claimed $L$. In Appendix A we provide an example that illustrates this point and shows how the Agent can use mixing to a higher effort level to monitor the Principal.

\section{Conclusions}

Relaxing the assumption that output is common knowledge, we were able to explain many features of the labor markets that were at odds with the literature. In particular, we have provided a rationale for the observed wage compression and the use of efficiency wages together with the threat of termination to provide incentives.

This information structure can also be used to better understand questions of organizational design and potentially the theory of the firm. In particular we described how having different 
hierarchies or setting up tournaments can be used to simultaneously provide incentives for the Agents to exert effort and for the principal to be truthful.

Additionally our paper provides a useful benchmark in which there is no need for the use of communication between Agents and principals. An important avenue for future research is to explore further possible reasons for the existence of communication. As shown in the appendix a possible solution might come from allowing the Agent to monitor the principal by randomizing his effort choice to inefficiently high effort levels. Alternatively, the existence of match-specific productivity parameters might provide a rationale for communication. We hope to address these issues in future research.

\section{References}

[1] Abreu, D., Milgrom, P. and Pearce, D. "Information and timing in Repeated Partnerships". Econometrica November 1991, 59(6), pp1713-33.

[2] Abreu, D., Pearce, D. and Stacchetti, E. "Toward a Theory of Discounted Repeated Games with Imperfect Monitoring". Econometrica, September 1990, 58(5), pp. 1041-63.

[3] Amarante, M. (2003) "Recursive Structure and Equilibria in Games with Private Monitoring" Economic Theory 22, 353-374 (2003)

[4] Aoyagi, M. "Collusion in dynamic Bertrand oligopoly with correlated private signals and communication", J. Econ. Theory 102 (2002), 229-248.

[5] Bhaskar,V. and Van Damme, E. 'Moral Hazard and Private monitoring' JET 1022002

[6] Baker, G., Gibbons, R. \& Murphy, K. J. (1994), 'Subjective performance measures in optimal incentive contracts', Quarterly Journal of Economics 109(4), 1125-1156.

[7] Baker, G., Gibbons, R. \& Murphy, K. J. (2002), 'Relational contracts and the theory of the firm', Quarterly Journal of Economics 117(1), 39-84.

[8] Baker, G.P. (1992), 'Incentive contracts and performance measurement', Journal of Political Economy 100(3), 598- 614 .

[9] Bewley, T. F. (1999), Why Wages Don’t Fall During a Recession, Harvard University Press, Cambridge, MA.

[10] Brown, M., Falk, A. \& Fehr, B. (2002), Contractual incompleteness and the nature of market imperfections, Discussion Paper 3272, CEPR. 
[11] Che, Y.-K. \& Yoo, S.-W. (2001), 'Optimal incentives for teams', American Economic Review $91(3), 525-541$.

[12] Compte, O. (1998) 'Comunication in Repeated Games with Imperfect Private Monitoring' Econometrica 66, pp. 597-626

[13] Compte,O. "On failing to cooperate when monitoring is private", J. Econ. Theory 102 (2002), $151-188$.

[14] Compte, O. "On sustaining cooperation without public observations", J. Econ. Theory 102 (2002), 106-150..

[15] Doornik, K. (2001), Relational contracting in partnerships. University of Oxford, Said Business School.

[16] Fehr, E. \& Falk, A. (2002), 'Psychological foundations of incentives', European Economic Review 46(4/5), 687-724.

[17] Fuchs, W. (2004) 'Subjective Evaluations: The Bonus as a signal of Performance'. Mimeo Stanford GSB.

[18] Garvey, G. T. (1995), 'Why reputation favors joint ventures over vertical and horizontal integration: A simple model', Journal of Economic Behavior and Organization 28(3), 387 - 397.

[19] Gibbons R.and Waldmann M..(1999) "Careers in Organizations: Theory and Evidence" Handbook of Labor Economics, Volume 3 Chapter 36.

[20] Holmstrom and Milgrom (1991). "Multitask Principal Agent Analyses: Incentive Contracts, Asset Ownership and Job Design". J. Law Econ. \& Organ. Vol 7 pp. 24-52

[21] Kahn, C. and D. Mookherjee (1998). "A Competitive Efficiency Wage Model with Keynesian Features" The Quaterly Journal of Economics 103(4) 609-645.

[22] Kandori,M "Introduction to Repeated Games with Private Monitoring" Journal of Economic Theory 102, 1-15 (2002)

[23] Kandori, M. and H. Matsushima, Private observation, communication and collusion, Econometrica 66 (1998), 627-652.

[24] Lazear, E. and S. Rosen, "Rank-Order Tournaments as Optimum Labor Contracts," Journal of Political Economy 1981.

[25] Lazear, E. (2004) "Output-based Pay: Incentives or Sorting? "Forthcoming, Research in Labor Economics, 2004. 
[26] Levin, J. (2002), 'Multilateral contracting and the employment relationship', Quarterly Journal ofEconomics 117(3), 1075-1103.

[27] Levin, J. (2003), 'Relational incentive contracts', American Economic Review. June 2003, 93(3) pp.835-57

[28] MacLeod, W. B. (2003), 'Optimal contracting with subjective evaluation', American Economic Review March 2003, 92(1) pp. 216-40

[29] MacLeod, W. B. \& Malcomson, J. M. (1989), 'Implicit contracts, incentive compatibility, and involuntary unemployment', Econometrica 57(2), 447-480.

[30] MacLeod, W. B. \& Parent, D. (1999), 'Jobs characteristics and the form of compensation', Research in Labor Economics 18, 177-242.

[31] Malcomson, J. M.(1984) "Work Incentives, hierarchy, and Internal Labour Markets", Journal of Political Economy 92: 486-507.

[32] Malcomson, J.M. (1999) "Individual Employment Contracts" Handbook of Labor Economics, Volume 3, Chapter 35.

[33] Millward, N., Stevens, M., Smart, D. \& Hawes, W. R. (1992), Workplace Industrial Relations in Transition. The ED/ESRC/PSL/ACAS Surveys, Dartmouth,Aldershot, UK.

[34] Pearce, D. \& Stacchetti, E. (1998), 'The Interaction of Implicit and Explicit Contracts in Repeated Agency', Games and Economic Behavior 23(1), 75-96.

[35] Prendergast, C. (1999) "The Provision of Incentives in Firms" Journal of Economic Literature, March 1999, 36(1), pp 7-63.

[36] Radner, R. (1985), 'Repeated principal-agent games with discounting', Econometrica 53(3), $1173-1198$.

[37] Rosen, "Prizes and Incentives in Elimination Tournaments," American Economic Review 1986.

[38] Sannikov, Y. (2004) "A Continuous-Time Version of the Principal-Agent Problem" Stanford GSB

[39] Shapiro, C. \& Stiglitz, J. (1984), 'Equilibrium Unemployment as a Worker Discipline Device', American Economic Review 74(3), 433-444. 


\section{Appendix A}

\subsection{No Mixing No Talking}

Consider the following one period review contract with random effort. The Agent plays $e=1$ with probability $\alpha \in(0,1)$, the Principal then observes the output and they simultaneously announce the actual effort and the actual output.

The following table summarizes the parameters (other than $\alpha$ ) of the self-enforcing contract conditional on the announcements.

\begin{tabular}{|c|c|c|}
\hline Announcements $(e, y)$ & Wage & Termination Probability \\
\hline$(0, L)$ & $w_{0 L}$ & $\beta_{0 L}$ \\
\hline$(0, H)$ & $w_{0 H}$ & $\beta_{0 H}$ \\
\hline$(1, L)$ & $w_{1 L}$ & $\beta_{1 L}$ \\
\hline$(1, H)$ & $w_{1 H}$ & $\beta_{1 H}$ \\
\hline
\end{tabular}

Continuation Values (including wages not including cost of effort)

\begin{tabular}{|c|c|c|}
\hline Principal & $y=L$ & $y=H$ \\
\hline$e=1$ & $F_{1 L}$ & $F_{1 H}$ \\
\hline$e=0$ & $F_{0 L}$ & $F_{0 H}$ \\
\hline
\end{tabular}

\begin{tabular}{|c|c|c|}
\hline Agent & $y=L$ & $y=H$ \\
\hline$e=1$ & $V_{1 L}$ & $V_{1 H}$ \\
\hline$e=0$ & $V_{0 L}$ & $V_{0 H}$ \\
\hline
\end{tabular}

In order to simplify our analysis and exposition, we definie the following new variables:

$$
\begin{aligned}
& \Delta_{1}=\alpha\left(F_{1 H}-F_{1 L}\right) \\
& \Delta_{0}=(1-\alpha)\left(F_{0 L}-F_{0 H}\right) \\
& \Delta_{L}=V_{1 L}-V_{0 L} \\
& \Delta_{H}=V_{1 H}-V_{0 H} \\
& \Delta_{\text {cross }}=V_{1 H}-V_{0 L}
\end{aligned}
$$

For the Principal we have the following truth-telling constraints:

Given that he observed $y=H$ :

$$
\Delta_{0} \leq \frac{p}{q} \Delta_{1}
$$

Given that he observed $y=L$ :

$$
\Delta_{0} \geq \frac{(1-p)}{(1-q)} \Delta_{1}
$$

We can represent these constraints graphically: 


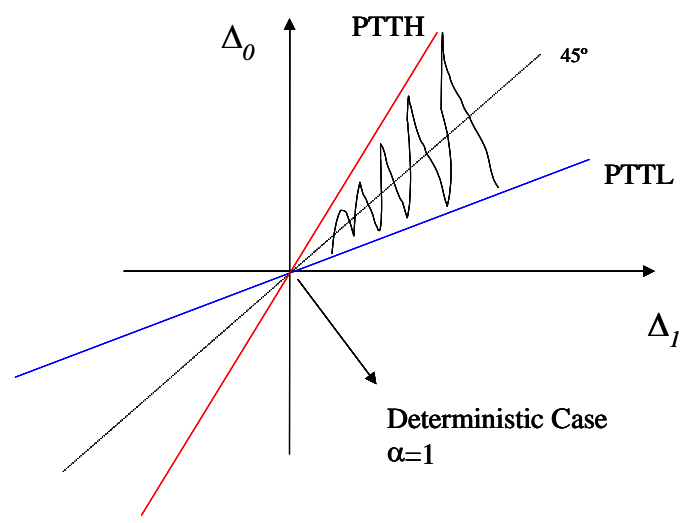

These constraints imply that $\Delta_{0}, \Delta_{1} \geq 0$

For the Agent we have the following two truth-telling constraints:

Given he exerted high effort:

$$
\Delta_{H} \geq-\frac{(1-p)}{p} \Delta_{L}
$$

Given he exerted low effort:

$$
\Delta_{H} \leq-\frac{q}{(1-q)} \Delta_{L}
$$

We also need the Agent to be indifferent between high and low effort

$$
\Delta_{H}=\frac{c-(p-q) \Delta_{\text {cross }}}{q}-\frac{(1-p)}{q} \Delta_{L}
$$

Graphically:

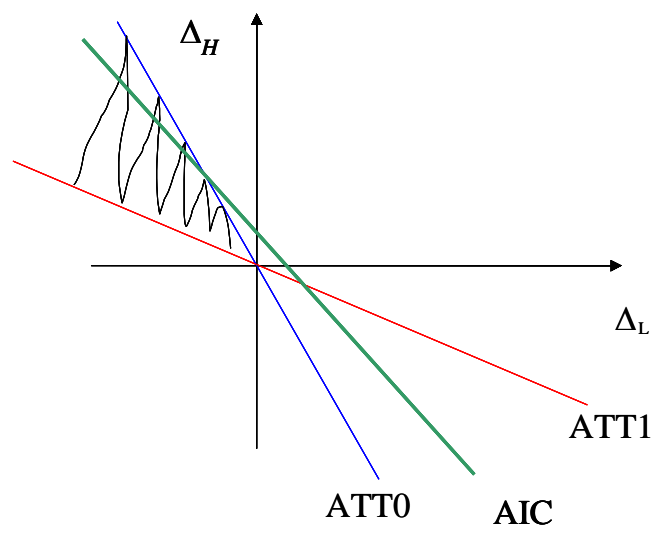

Note that $(A I C)$ has always a slope between $(A T T 0)$ and $(A T T 1)$. 
With these preliminaries, we now prove Proposition13 from the text.

Proposition 13 Allowing for contracts in which the Agent randomizes and the continuation values are determined as a function of the simultaneous announcements by the Agent and the Principal does not provide any improvement over the one-period review contract in which the Agent exerts high effort deterministically.

Proof. Suppose that the optimal contract has $\alpha<1$ and $\Delta_{1}$ and $\Delta_{0}>0$ and that $(P T T H)$ does not bind. Now consider the following: decrease $w_{1 L}$ and $\beta_{1 L}$ and increase $w_{1 H}$. We can do this such a way that the resulting increase in $V_{1 H}$ is equal to $\frac{1-p}{p}$ times the decrease in $V_{1 L}$. As a result, the Agent's IC constraints continue to hold and the truthtelling constraints are relaxed. The decrease in $\beta_{1 L}$ implies an improvement in efficiency since there will be less value burning. Therefore, $($ PTTH must be binding in the optimal contract.

Now suppose that $(P T T H)$ binds, but that $(P T T L)$ does not bind. If we could decrease. $\Delta_{0}$ we would relax $(P T T H)$ and improve. This can be achieved by simply increasing $\alpha$. Changing $\alpha$ has no impact on the Agent's constraints, it relaxes (PTTH) and we can do it till (PTTL) starts binding. If we iterate between this two steps (see graph below) clearly we can drive $\Delta_{1}$ and $\Delta_{0}$ all the way to zero. This means that either $\alpha=1$ or that $\left(F_{0 L}-F_{0 H}\right)=\left(F_{1 H}-F_{1 L}\right)=0$ but if this is the case we can set $\alpha=1$ anyway and this is preferable since lower $\alpha^{\prime} s$ implies an efficiency loss.

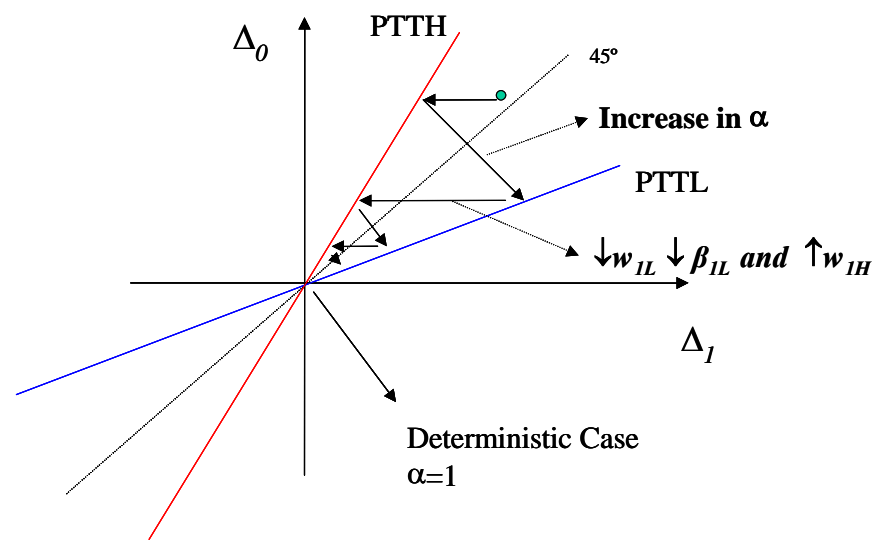

Mixing by the Agent fails as means to relax the Principal's truthtelling constraints when he observes a high realization of outcome. The intuition behind this negative result is that when the Agent mixes the Principal is actually getting some slack from claiming $y=L$ when $y=H$. This is because there is some chance that the Agent had actually done low effort and the Principal cannot be punished for claiming $L$ when no effort effort was exerted. If instead we allowed the Agent to mix a higher effort level (even if it were inneficient from a productive perspective) then mixing might work. We illustrate this with an example in the next section. 


\subsection{Monitoring with high Effort}

Suppose that the Agent could additionally choose to exert a higher effort level. If this effort level leads to high outcome with higher probability, mixing by the Agent to this higher effort level relaxes the Principal's truth-telling constraints. The intuition for this is clearer when we consider the case in which very high effort leads to a high output for sure. Suppose the Agent mixes between these two effort levels, when the Principal observes high output he is now less tempted to claim low. He fears that the Agent might have exerted very high effort and that he would be caught lying if he did.

To illustrate more precisely how this works consider the following example: let $e=\{l, m, h\}$ and $c(e)=\{0, c, 1\}$ with $p(e)=\{0, p, 1\}$ respectively. ${ }^{28} H=1$ and $L=0$. Medium effort is the only productively efficient effort level $p-c>0$.

Suppose we wish to have the Agent mix between high and medium effort and we allow continuation values including wages to depend on the simultaneous announcements. Denote these by $V_{\tilde{e}, \tilde{y}}$ for the Agent and $F_{\tilde{e}, \tilde{y}}$ for the Principal. We face the following constraints:

For the Agent:

Thruthtelling:

Given he exerted $m$ effort:

$$
p V_{m H}+(1-p) V_{m L} \geq p V_{h H}+(1-p) V_{h L}
$$

Given he exerted $h$ effort:

$$
V_{h H} \geq V_{m H}
$$

Incentive compatibility for mixing:

$$
p V_{m H}+(1-p) V_{m L}-c=V_{h H}-1 \geq V_{m L}
$$

For the Principal:

Thruthtelling:

Given he observed $L$ :

$$
F_{m L} \geq F_{m H}
$$

\footnotetext{
${ }^{28}$ Similar examples could be constructed for less extreme cases, we pick these parameters for expositional convenience.
} 
Given he observed $H$ :

$$
\alpha F_{h H}+(1-\alpha) p F_{m H} \geq \alpha F_{h L}+(1-\alpha) p F_{m L}
$$

Consider a contract in which the Principal gets all the residual surplus.

\begin{tabular}{|c|c|c|}
\hline Announcements & Wage & Termination Probability \\
\hline$(m, L)$ & $w_{m L}=0$ & $\beta_{m L}=0$ \\
\hline$(m, H)$ & $w_{m H}=\frac{c}{p}$ & $\beta_{m H}=0$ \\
\hline$(h, L)$ & $w_{h L}=-\frac{p}{1-p}$ & $\beta_{h L}=1$ \\
\hline$(h, H)$ & $w_{h H}=1$ & $\beta_{h H}=0$ \\
\hline
\end{tabular}

The Agent has no incentives to deviate since when he is called to exert high effort he is compensated for sure with the cost he had to bare. When he is called to do medium effort he gets zero if he obeys. If he chooses to exert low effort the best he can get is also a payoff of zero.

We must now make sure that the Principal does not have incentives to claim $L$ when he observes $H .{ }^{29}$ Using the table above we can re-write $(T T H)$ as follows:

$$
\begin{aligned}
& \alpha\left(-1+\frac{\delta((1-\alpha)(p-c))}{1-\delta}\right)+(1-\alpha) p\left(-\frac{c}{p}+\frac{\delta((1-\alpha)(p-c))}{1-\delta}\right) \\
\geq & \alpha \frac{p}{1-p}+(1-\alpha) p\left(\frac{\delta((1-\alpha)(p-c))}{1-\delta}\right)
\end{aligned}
$$

We will use the following parameter values to illustrate the example: $p=\frac{1}{2}, \delta=.9$ and $c=\frac{1}{8}$. Using these particular values and doing some algebra, we see that satisfying $(T T H)$ reduces to finding an $\alpha$ that satisfies:

$$
-\frac{27}{4} \alpha^{2}+3 \alpha-\frac{1}{4} \geq 0
$$

Any $\alpha \in\left[\frac{1}{9}, \frac{1}{3}\right]$ is a solution to this, but since $m$ is more productive than $h$, it is optimal to pick $\alpha=\frac{1}{9}$. In this case we get a total expected surplus of $3 \frac{1}{3}$. The First Best surplus is $\frac{15}{4}=3.75$ and with the deterministic one-period review contract using our results from Section 5.1.1 we get an expected surplus of only $\frac{2}{3} F B=2.5$. This example shows that when considering a more general model, with many possible effort choices, ruling out mixed strategies by the Agent and communication might reduce efficiency.

\footnotetext{
${ }^{29}$ It is easily verified that $(T T L)$ is satisfied.
} 


\section{Appendix B}

Proof of Proposition 1. Follows directly from Lemmas (5) and (6) stated below. Adding equations (4) and (5) delivers (1).

Lemma 5 The ICA implies:

$$
\begin{aligned}
& \mathbb{E}\left[V_{t}^{+}\left(\left\{h_{t-1}^{P}, y_{t}=H\right\}, \sigma\right) \mid\left\{h_{t-1}^{A}, e_{t}=1\right\}, \sigma\right] \\
> & \mathbb{E}\left[V_{t}^{+}\left(\left\{h_{t-1}^{P}, y_{t}=L\right\}, \sigma\right) \mid\left\{h_{t-1}^{A}, e_{t}=1\right\}, \sigma\right],
\end{aligned}
$$

i.e. the expected payoff to the Agent is strictly increasing in the output when the Agent is supposed to exert effort.

Proof. Follows from the fact that effort increases the likelihood of $y_{t}=H$.

Lemma 6 The IC for the Principal implies:

$$
F_{t}^{+}\left(\left\{h_{t-1}^{P}, y_{t}=H\right\}, \sigma\right)=F_{t}^{+}\left(\left\{h_{t-1}^{P}, y_{t}=L\right\}, \sigma\right) \quad \forall h_{t-1}^{P}
$$

Proof. Suppose first that for some $h_{t-1}^{P}$ :

$$
F_{t}^{+}\left(\left\{h_{t-1}^{P}, y_{t}=H\right\}, \sigma\right)>F_{t}^{+}\left(\left\{h_{t-1}^{P}, y_{t}=L\right\}, \sigma\right),
$$

and that $y_{t}=L$ is realized.

Now, consider the alternative strategy $\tilde{\sigma}_{\omega}^{P}$ for the Principal. $\tilde{\sigma}_{\omega}^{P}$ follows $\sigma_{\omega}^{P}$ except that it implies the same actions as $\sigma_{\omega}^{P}$ for histories with $y_{t}=L$ as it does for histories where $y_{t}=H$. This deviation is undetectable by the Agent and leads to an improvement for the Principal. ${ }^{30}$

A similar argument can be constructed if:

$$
F_{t}^{+}\left(\left\{h_{t-1}^{P}, y_{t}=H\right\}, \sigma\right)<F_{t}^{+}\left(\left\{h_{t-1}^{P}, y_{t}=L\right\}, \sigma\right) .
$$

Therefore, it must be the case that

$$
F_{t}^{+}\left(\left\{h_{t-1}^{P}, y_{t}=H\right\}, \sigma\right)=F_{t}^{+}\left(\left\{h_{t-1}^{P}, y_{t}=L\right\}, \sigma\right),
$$

for all $h_{t-1}^{P}$.

Lemmas 5 and 6 imply that for any strategy pair $\sigma^{*}$ and history $h_{t-1}^{V}$ such that $\sigma^{*}$ induces $e\left(h_{t-1}^{V}\right)=1$, the Agent's continuation value $v_{t}^{+}$must depend on the outcome but the Principal's continuation value $F_{t}^{+}$must be equal after either outcome. This implies that the total continuation surplus depends on the outcome realization.

\footnotetext{
${ }^{30}$ For the case when $p=1$ and $q>0$. We have to be careful when $e_{t}=1$ because now the Agent can detect a deviation if the Principal claims $y_{t}=L$.
} 
Lemma 7 Given $\sigma^{A}$ and $\omega$ if for all $\left\{h_{t-1}^{P}, y_{t}\right\} \sigma_{\omega}^{P}$ is incentive compatible for the Principal then $\sigma_{\omega}^{P}$ is a best response to $\sigma^{A}$ given $\omega$.

Proof. Follows directly from the definition of best response. There is no $\tilde{\sigma}_{\omega}^{P} \in \Sigma_{\omega}^{P}$ that can achieve a higher value for the Principal.

Technically, we are abusing the definition of best response slightly because we are not explicitly considering detectable deviations. On the other hand these deviations could be delt with in a simple way.

\section{Restatement of Theorem 1}

Given any contract $\left(\omega, \sigma_{\omega}\right)$ that generates values $V$ and $F$, we can construct a payoff equivalent contract $\left(\hat{\omega}, \hat{\sigma}_{\omega}\right)$ with the following properties:

(i) The Agent receives a constant wage until he is fired and no bonuses.

(ii) The Agent exerts effort every period until he is fired.

(iii) The Principal gives no feedback (sends no messages) to the Agent.

Proof. Properties (i) and (ii) follow from applying successively Lemmas (8), (9) and (10). Given properties (i) and (ii) Lemma (11) delivers property (iii).

Lemma 8 For any contract $\left[\omega, \sigma^{*}\right]$ that generates values $V$ and $F$ there is a payoff-equivalent contract $\left[\tilde{\omega}, \tilde{\sigma}^{*}\right]$ such that for any history $h_{t-1}^{V}$ with the property that $e\left(h_{t-1}^{V}\right)=0$ all future actions are independent of the outcome $y_{t}$.

Proof. Suppose the Principal conditioned his action on $y_{t}$. Now, instead let him condition his strategy $\tilde{\sigma}^{P}$ on $\phi_{t}$ in the following way: if $\phi_{t}<q$ let the Principal take the same actions that he did for $\sigma^{P}$ when $y_{t}=H$ and if $\phi_{t}>q$ take the same actions he did for $\sigma^{P}$ when $y_{t}=L$. The Principal had to be indifferent between the outcomes of $y_{t}$ by Lemma 6 hence he will not have incentives to deviate from $\tilde{\sigma}$ if he didn't have incentives to deviate from $\sigma$. If the Agent follows the equilibrium strategy, his payoffs and information are the same as in the original equilibrium. Therefore, if he follows $e\left(h_{t-1}^{V}\right)=0$ the incentives to deviate in later or earlier periods are unchanged. If he deviates in the current period to $e\left(h_{t-1}^{V}\right)=1$ he is not affecting future payoffs nor obtaining any information hence such deviation costs him $c$ without any benefit.

In words, to induce no effort we don't need to provide any incentives to the Agent. Therefore, we can always make his payoff unconditional of the outcome without inducing him to put the effort.

Definition 7 A contract $\left[\omega, \sigma^{*}\right]$ is an input based compensation contract if $b_{t}+w_{t}=E\left[y_{t} \mid e_{t}\right]$ for all $t>$ 0 
Lemma 9 (Input based pay) For every contract $\left[\omega, \sigma^{*}\right]$ that generates values $V$ and $F$ there exists a payoff equivalent input based compensation contract $\left[\tilde{\omega}, \tilde{\sigma}^{*}\right]$.

Proof. Modify $\left[\omega, \sigma^{*}\right]$ in the following way: let $\tilde{b}_{t}+\tilde{w}_{t}=E\left[y_{t} \mid e_{t}\right]$ for all $t>0$ and $\tilde{w}_{0}=$ $E\left[y_{0} \mid e_{0}\right]-F$. Also, let $\tilde{a}_{t}=a_{t} k_{t}$ and $\tilde{k}_{t}=1 \forall t$. This changes imply that the Principal gets $F$ as in the initial contract but also, that for all $t$ the Principal's continuation value is zero (equal to his outside option). Therefore, there are no incentive issues having the Principal, who is indifferent on whether to terminate or not, do all the termination. Additionally, there are no incentive problems regarding the principal's announcements. By construction, his announcements might change effort level and compensation, but they do not change his expected surplus. The Agent won't want to deviate with respect to $\{\tilde{k}\}$ since terminating when he is not supposed to, would make him worse off as he gets all the surplus in the continuation game. Lemma 8 implies we only need to check that there will be no deviations with respect to effort for those histories where effort is supposed to be exerted. Note that under the original contract, when the Agent was supposed to exert effort there had to be value burning in case of a bad outcome to ensure that the right incentives were provided for the Agent to exert effort. We showed already that the new contract will destroy the same amount of value after the same histories than the original contract, hence the Agent will have the same incentives to exert effort under the new contract as he had under the old.

Definition 8 A contract $\left[\omega, \sigma^{*}\right]$ is a termination contract if it is an input based compensation contract and the Agent exerts effort every period until termination.

Lemma 10 (Termination) For any input based compensation contract $\left[\omega, \sigma^{*}\right]$ that generates values $V$ and $F$ there is a payoff equivalent termination contract $\left[\tilde{\omega}, \tilde{\sigma}^{*}\right]$.

Proof. First we will use Lemma 8 to transform $\left[\omega, \sigma^{*}\right]$ into $\left[\hat{\omega}, \hat{\sigma}^{*}\right]$. Note that this implies that the destruction of value that is produced by having the Agent exert no effort after history $h_{t-1}^{V}$ for a period is independent of $y_{t}$. When there is a period of no effort, value is being destroyed because there is a delay of one period until any future surplus is realized. Therefore if we let $\tilde{a}\left(h_{t-2}^{P}, y_{t}, x_{t}\right)=\hat{a}\left(h_{t-2}^{P}, y_{t}, x_{t}\right) \delta$ we destroy in expectation the same amount of value. Finally, we must adjust all strategies by one period since we are eliminating the slack period.

This shows that incentives for the Agent to exert effort can be provided via efficiency wages and the threat of termination.

Definition 9 A termination contract $\left[\omega, \sigma^{*}\right]$ is a no communication contract iff the messages $m_{t}$ are completely uninformative $\forall t$.

Lemma 11 (No communication) For any termination contract $\left[\omega, \sigma^{*}\right]$ there is a payoff equivalent no communication contract $\left[\tilde{\omega}, \tilde{\sigma}^{*}\right]$. 
Proof. First note that in a termination contract on the equilibrium path the Agent's actions are independent of the messages $m_{t}$. Having the Agent completely uninformed does not give the Principal any profitable deviation possibilities so he will still follow the original termination rule $\{a\}$. Finally, if for every message $m_{t}$ that the Agent could have received, he chose to exert effort that means that if now he has no message on which to condition his action he would still choose to exert effort.

Proof of Proposition 2. When $T=1$, since this is the last period of the game, the continuation values for both, the Agent and the Principal, solely consists on the current compensation. By Lemma 6 the total compensation the Principal pays must be the same for either realization of output. Hence, providing incentives for the Agent to exert effort, which requires compensation to be dependent on the output, is not possible.

Next consider any arbitrary finite horizon. Suppose there exists a contract that contemplates the Agent exerting effort in some periods. Consider the last period in which the Agent is supposed to exert effort. By the arguments given to prove the case $T=1$ it follows that incentives cannot be provided to exert effort for this last period hence contradicting the claim that there can be a contract for finite $T$ in which effort is exerted.

Proof of Lemma 1. Suppose that after some history $h_{t-1}^{V} \times m^{t} \times[0,1]$ a payment $w_{t}^{p}$ was to be made by the Principal and $w_{t}^{A} \leq w_{t}^{P}$ to be received by the Agent. We can simply have at time $T$ the Principal paying $\frac{w_{t}^{p}}{\delta^{T-t}}$ and the Agent receiving $\frac{w_{t}^{A}}{\delta^{T-t}}$ with the difference, if any, being burnt at time $T$.

\section{Proof of Lemma 2.}

Follows from Lemma (10) and noting that the destruction of value that can be achieved by termination can also be achieved by burning money.

Proof of Proposition 3. The proof to the first part of the Proposition is organized in four steps. First we write the problem, then we write a relaxed problem where only one-step deviations by the Agent are considered. The third step solves the relaxed problem and the last step proves that this is also a solution to the original problem.

i) To motivate effort for $T+1$ periods which we denote $\mathbf{e}^{*}$, we face the following money burning minimization problem:

$$
\begin{gathered}
\min _{\left\{Z\left(y^{T}\right)\right\} y^{T} \in Y^{T}} \delta^{T} \sum_{y^{t} \in Y^{T}} Z\left(y^{T}\right) P\left(y^{T} \mid \mathbf{e}^{*}\right) \\
\text { s.t. } \quad \mathbf{c}\left(\mathbf{e}^{*}-\mathbf{e}\right)+\delta^{T} \sum_{y^{T} \in Y^{T}} Z\left(y^{T}\right)\left(P\left(y^{T} \mid \mathbf{e}^{*}\right)-P\left(y^{T} \mid \mathbf{e}\right)\right) \leq 0 \quad \forall \mathbf{e} \in\{0,1\}^{T+1}
\end{gathered}
$$




$$
Z\left(y^{T}\right) \geq 0 \quad \forall y^{T}
$$

Note that the Principal's incentive constraints are satified by construction since $w^{P}$ is fixed.

ii) Instead of solving this problem directly, we first solve a relaxed problem in which we only consider one-step deviations by the Agent:

$$
\begin{gathered}
\min _{\left\{Z\left(y^{T}\right)\right\} y^{T} \in Y^{T}} \delta^{T} \sum_{y^{t} \in Y^{T}} Z\left(y^{T}\right) P\left(y^{T} \mid \mathbf{e}^{*}\right) \\
\text { s.t. } \quad \delta^{t} c+\delta^{T} \sum_{y^{T} \in Y^{T}} Z\left(y^{T}\right) P\left(y^{T} \mid \mathbf{e}^{*}\right)\left(\frac{P\left(y_{t} \mid e_{t}=1\right)-P\left(y_{t} \mid e_{t}=0\right)}{P\left(y_{t} \mid e_{t}=1\right)}\right) \leq 0 \quad \forall t \\
Z\left(y^{T}\right) \geq 0 \quad \forall y^{T} .
\end{gathered}
$$

From (7) it is clear that in order to provide incentives in period $t$, we need:

$$
\left(\frac{P\left(y_{t} \mid e_{t}=1\right)-P\left(y_{t} \mid e_{t}=0\right)}{P\left(y_{t} \mid e_{t}=1\right)}\right)<0 .
$$

In words, to provide incentives for effort in period t we need to have burning if the outcome of that period is low which, is more likely to occur if the Agent deviates. Therefore, the constraints can be written as:

$$
\delta^{t} c \frac{(1-p)}{p-q} \leq \delta^{T} \sum_{y^{T} \in Y^{T} \cap\left(y_{t}=L\right)} Z\left(y^{T}\right) P\left(y^{T} \mid \mathbf{e}^{*}\right) \quad \forall t
$$

Clearly $t=0$ is the most binding constraint if $\delta<1$ which puts a lower bound on the necessary expected money burning $c \frac{(1-p)}{(p-q)}$.

iii) Next we show that:

$$
Z^{*}=\left\{\begin{array}{ll}
\frac{c}{\delta^{T}(p-q)(1-p)^{T}} & \text { if } y^{T}=\mathbf{L} \\
0 & \text { otherwise }
\end{array}\right\}
$$

achieves the lower bound and satisfies all the IC constraints of the relaxed problem.

First note that:

$$
P\left(y^{T}=\mathbf{L} \mid \mathbf{e}^{*}\right)=(1-p)^{T+1} .
$$

Hence,

$$
\delta^{T} \sum_{y^{T} \in Y^{T}} Z^{*}\left(y^{T}\right) P\left(y^{T} \mid \mathbf{e}^{*}\right)=c \frac{(1-p)}{(p-q)} \geq \delta^{t} c \frac{(1-p)}{(p-q)} \quad \forall t
$$

iv) We now show that $Z^{*}$ is also a solution to the original problem.

Using $Z^{*}$, the constraints of the original problem (6) take the following form: 


$$
\text { c. }\left(\mathbf{e}^{*}-\mathbf{e}\right)+\frac{c}{(p-q)(1-p)^{T}}\left((1-p)^{T+1}-P\left(y^{T} \mid \mathbf{e}\right)\right) \leq 0 \quad \forall \mathbf{e} \in\{0,1\}^{T+1} .
$$

Letting $n$ denote the number of periods the Agent deviates we can write the term that captures the expected cost of deviation as follows:

$$
\frac{c}{(p-q)(1-p)^{T}}\left((1-p)^{T+1}-(1-p)^{(T+1)-n}(1-q)^{n}\right)
$$

Now suppose the Agent was deviating in $n-1$ periods and now deviates for $n$ periods. The increase in the probability that $y^{T}=\mathbf{L}$ is:

$$
\left(\frac{1-q}{1-p}\right)^{n-1}(1-p)^{T}(p-q) .
$$

Since $\left(\frac{1-q}{1-p}\right)>1$ the increase in the probability of punishment from an additional deviation is increasing in the number of previous deviations. Hence, if the Agent doesn't find it profitable to deviate once, he won't want to deviate at all. Given $\delta<1$ and $Z^{*}$, the most profitable single deviation is in the first period. This is not profitable by step (iii).

Finally, the conditions for $w^{P}$ guarantee that individual rationality constraints are satisfied so the players sign the contract $\omega$ at time zero.

Uniqueness proof for the case when $\delta=1$ :

Consider any other incentive compatible contract $Z$. Clearly, for $Z$ to be optimal it must be that $Z\left(y^{T}=\mathbf{L}\right)<Z^{*}\left(y^{T}=\mathbf{L}\right)$ otherwise $Z^{*}$ would imply strictly less expected money burnt. Denote all histories $y^{T} \neq \mathbf{L}$ for which $Z\left(y^{T}\right)>0$ and $y_{0}=L$ by $\dot{y}^{T}$. If there is no such history, then $Z\left(y^{T}=\mathbf{L}\right) \geq Z^{*}\left(y^{T}=\mathbf{L}\right)$ otherwise, the Agent would deviate in the first period. Hence, if $Z$ is to be optimal it must be that there is some $\dot{y}^{T}$. Suppose there is one such $\dot{y}^{T}$. Let $j$ $=\min \left\{t: \dot{y}_{t}=H\right\}$. The incentive constraint for period $j$ effort is given by:

$$
\delta^{j} c+\delta^{T}\left(Z\left(y^{T}=\mathbf{L}\right)(1-p)^{T+1}\left(\frac{q-p}{1-p}\right)+Z\left(\dot{y}^{T}\right) P\left(\dot{y}^{T}\right)\left(\frac{p-q}{p}\right)\right) \leq 0
$$

Now, let $\delta \rightarrow 1$

$$
c+Z\left(y^{T}=\mathbf{L}\right)(1-p)^{T+1}\left(\frac{q-p}{1-p}\right)+Z\left(\dot{y}^{T}\right) P\left(\dot{y}^{T}\right)\left(\frac{p-q}{p}\right) \leq 0
$$

This is the IC for period 0 effort. Note this implies that $Z$ cannot be incentive compatible since this constraint held with equality for $Z^{*}$ and $Z\left(y^{T}=\mathbf{L}\right)<Z^{*}\left(y^{T}=\mathbf{L}\right)$ and additionally, the last term in the left hand side of the inequality is positive. Therefore, $Z^{*}$ is the only contract that is optimal for all $\delta$. 


\section{Proof of Proposition 4 .}

All of the Agent's IC constraints are holding with equality when he is completely uninformed. As soon as he can condition on any information, the probability he assigns to his effort being pivotal in any given period would change. Some IC constraints would therefore be violated when he updates this probability given the messages.

Proof of Lemma 4. It follows from noting that as $\delta \rightarrow 1$ and rearranging (2) becomes $s>$ $\frac{c(1-p)}{(p-q)} \frac{1}{T}$. Since the left hand side is a positive number we can always find a large enough $T$ so that the condition is satisfied. Note as well that for any fixed $T$ as $\delta \rightarrow 1, \beta \rightarrow 0$ so there is a feasible $\beta^{*}$ to implement the contract.

Proof of Theorem 2 (Folk Theorem). The inefficiency $\lambda$ is given by:

$$
\lambda_{T}=\frac{c(1-p)}{(p-q)} \frac{1}{S_{T}}
$$

Taking the limit as $\delta \rightarrow 1$

$$
\lim _{\delta \rightarrow 1} \lambda_{T}=\frac{c(1-p)}{(p-q) T(\varepsilon, s) s}
$$

Now let $T(\varepsilon, s)$ be greater than $\bar{T}$, where:

$$
\bar{T}=\frac{c(1-p)}{\varepsilon(p-q) s}
$$

Therefore the inefficiency is:

$$
\lambda_{T}=\frac{c(1-p)}{T(\varepsilon, s)(p-q) s}<\frac{c(1-p)}{\frac{c(1-p)}{\varepsilon(p-q) s}(p-q) s}=\varepsilon
$$

Restatement of Proposition 5 A sequence of termination rules $\left\{a\left(h^{t}\right)\right\}_{t=0}^{\infty}$ that does not have each of the following properties, can be weakly improved upon.

(i) For all $h^{t}$, if $a\left(h^{t}, H\right)<1$ then $a\left(h^{t}, L\right)=0$.

(ii) For all $h^{t}, a\left(h^{t}, L\right) \leq a\left(h^{t}, H\right)$.

(iii) For all $h^{t} \times h^{j}$, if $a\left(h^{t}, H, h^{j}\right)<1$ then $a\left(h^{t}, L, h^{j}\right)=0$.

(iv) For all $h^{t} \times h^{j}, f a\left(h^{t}, H, h^{j}\right) \geq a\left(h^{t}, L, h^{j}\right)$.

(v) For all $t$, if $a\left(L^{t}\right)>0$ then $a\left(h^{t}\right)=1$ for all $h^{t}$.

(vi) For all $h^{t}, V\left(h^{t}, H\right) \geq V\left(h^{t}, L\right)$.

(vii) For all $h^{t}$, if $a\left(h^{t}, L\right)=a\left(h^{t}, H\right)=0$ then $a\left(h^{t}\right)=0$.

Proof. (i) Suppose $a\left(h^{t}, H\right)<1$ and $a\left(h^{t}, L\right)>0$ for some $h^{t}$. Let $\tilde{a}\left(h^{t}, L\right)=a\left(h^{t}, L\right)-\varepsilon$ $\geq 0$ for some small $\varepsilon>0$. Also, instead of terminating with probability $1-a\left(h^{t}, H\right)$, after 
history $\left(h^{t}, H\right)$. Let the Principal terminate with probability $1-a\left(h^{t}, H\right)-\left(\frac{1-p}{p}\right) \varepsilon \geq 0$ and with probability $\left(\frac{1-p}{p}\right) \varepsilon$ switch for history $\left(h^{t}, L\right)$ upon observing the high outcome at $t+1$. That is, after history $\left(h^{t}, H\right)$ start the Agent at $t+2$ with history $\left(h^{t}, L\right)$ with probability $\left(\frac{1-p}{p}\right) \varepsilon$. We must make sure these changes respect two constrains: first the expected values at time $t$ must not be changed. This guarantees that incentives for all periods prior to $t+1$ are unaffected. Formally:

$$
\begin{aligned}
& p \times a\left(h^{t}, H\right) \times V\left(h^{t}, H\right)+(1-p) \times a\left(h^{t}, L\right) \times V\left(h^{t}, L\right) \\
= & p\left(a\left(h^{t}, H\right) \times V\left(h^{t}, H\right)+\left(\frac{1-p}{p}\right) \varepsilon V\left(h^{t}, L\right)\right)+(1-p) \times\left(a\left(h^{t}, L\right)-\varepsilon\right) \times V\left(h^{t}, L\right)
\end{aligned}
$$

Second, the probability distribution on the tree is unaffected so all incentive constraints for periods after $t+1$ are unaffected.

$$
(1-p) \times\left(a\left(h^{t}, L\right)-\varepsilon\right)+p\left(\frac{1-p}{p}\right) \varepsilon=(1-p) \times a\left(h^{t}, L\right)
$$

The key is that incentive constraints at period $t+1$ have been relaxed. Since the change has increased $V\left(h^{t}, H\right)$ and decreased $V\left(h^{t}, L\right)$ the Agent now has more incentives to exert effort at $t+1$.

(ii) Follows directly from (i) and the fact that $a\left(h^{t}\right) \in[0,1]$

(iii) Suppose $\exists h^{t}, h^{j}$ s.t. $a\left(h^{t}, H, h^{j}\right)<1$ and $a\left(h^{t}, L, h^{j}\right)>0$ and consider the following changes to $\left\{a\left(h^{t}\right)\right\}$. Reduce the termination probability after history $\left(h^{t}, H, h^{j}\right)$ to $1-a\left(h^{t}, H, h^{j}\right)-\varepsilon$ where $\varepsilon>0$. With probability $\varepsilon$ instead of terminating assume the history is $\left(h^{t}, L, h^{j}\right)$ and treat the Agent accordingly. Let $\tilde{a}\left(h^{t}, L, h^{j}\right)$ be the new continuation probability after $\left(h^{t}, L, h^{j}\right)$. Before the changes, the probability an Agent was hired to begin work in period $t+j+2$ with history $\left(h^{t}, L, h^{j}\right)$ was:

$$
P\left(h^{t}, L, h^{j}\right)\left(\prod_{\varnothing \leq h^{u} \leq\left(h^{t}, L, h^{j}\right)} a\left(h^{u}\right)\right)
$$

Now that probability is:

$$
\begin{aligned}
& P\left(h^{t}, L, h^{j}\right)\left(\prod_{\varnothing \leq h^{u}<\left(h^{t}, L, h^{j}\right)} a\left(h^{u}\right) \times \tilde{a}\left(h^{t}, L, h^{j}\right)\right) \\
& +P\left(h^{t}, H, h^{j}\right)\left(\prod_{\varnothing \leq h^{u}<\left(h^{t}, H, h^{j}\right)} a\left(h^{u}\right)\right) \times \varepsilon \times \tilde{a}\left(h^{t}, L, h^{j}\right) \\
= & \tilde{a}\left(h^{t}, L, h^{j}\right) P\left(h^{t}, L, h^{j}\right)\left(\prod_{\varnothing \leq h^{u}<\left(h^{t}, L, h^{j}\right)} a\left(h^{u}\right)+\varepsilon \frac{p}{1-p}\left(\prod_{\varnothing \leq h^{u}<\left(h^{t}, H, h^{j}\right)} a\left(h^{u}\right)\right)\right)
\end{aligned}
$$

This probability must be kept constant to guarantee incentive constraints in the future hold hence: 


$$
\tilde{a}\left(h^{t}, L, h^{j}\right)=a\left(h^{t}, L, h^{j}\right) \frac{\left(\prod_{\varnothing \leq h^{u}<\left(h^{t}, L, h^{j}\right)} a\left(h^{u}\right)\right)}{\left(\prod_{\varnothing \leq h^{u}<\left(h^{t}, L, h^{j}\right)} a\left(h^{u}\right)+\varepsilon \frac{p}{1-p}\left(\prod_{\varnothing \leq h^{u}<\left(h^{t}, H, h^{j}\right)} a\left(h^{u}\right)\right)\right)}
$$

This choice of $\tilde{a}\left(h^{t}, L, h^{j}\right)$ also leaves the continuation value $V\left(h^{t}\right)$ constant assuring that IC constraints for $\tau \leq t$ are unaffected. Incentive constraints between $t+2$ and $t+j+1$ are not affected either since the changes realized are only contingent on period $t+1$ action.

Finally, $V\left(h^{t}, H\right)$ has been increased and $V\left(h^{t}, L\right)$ decreased as a result of these changes so incentives in period $t+1$ have been relaxed. This implies at least a weak improvement can be achieved.

(iv) \& (v) Follow from the proof of (iii)

(vi) Since $\forall h^{t} \times h^{j} a\left(h^{t}, H, h^{j}\right) \geq a\left(h^{t}, L, h^{j}\right)$ then since:

$$
V\left(h^{t}\right)=a\left(h^{t}\right) \sum_{j=1}^{\infty} s\left(\delta^{j}\left(\sum_{h^{j}} P\left(h^{t}, h^{j}\right) \prod_{h^{\tau} \in\left(h^{t}, h^{j}\right)} a\left(h^{\tau}\right)\right)\right)
$$

it follows that $V\left(h^{t}, H\right) \geq V\left(h^{t}, L\right)$.

(vii) Suppose $a\left(h^{t}, L\right)=a\left(h^{t}, H\right)=0$ and $a\left(h^{t}\right)>0$. Let $\tilde{a}\left(h^{t}\right)=a\left(h^{t}\right)-\varepsilon$ for some small $\varepsilon>0$. Also with probability $\gamma\left(\tilde{h}^{t}\right)$ after $h^{t}$ let the Principal pretend that all other histories $\tilde{h}^{t}$ occurred where the relative weights are given according to the likelihood of each alternative history $\tilde{h}^{t}$ occurring on the equilibrium path. $\gamma$ must also be chosen so that the value after $h^{t}$ is kept constant. This change has kept values constant so past IC are still satisfied. The only change that we have made is to increase the probability that when deciding effort at $t+1$ the Agent is somewhere in the outcome tree where the current outcome realization affects his continuation value. Therefore, the incentive constraint for effort at $t+1$ has been relaxed. Incentives in all future periods are unaffected since the Agent's beliefs are the same that under the original contract. 